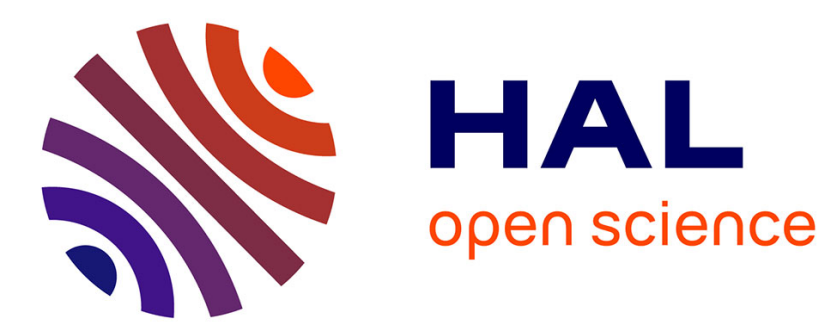

\title{
From interferometry to color holography
}

Jean-Michel Desse

\section{To cite this version:}

Jean-Michel Desse. From interferometry to color holography. Experimental Aerodynamics, 2017. hal-01633484

\section{HAL Id: hal-01633484 https://hal.science/hal-01633484}

Submitted on 13 Nov 2017

HAL is a multi-disciplinary open access archive for the deposit and dissemination of scientific research documents, whether they are published or not. The documents may come from teaching and research institutions in France or abroad, or from public or private research centers.
L'archive ouverte pluridisciplinaire HAL, est destinée au dépôt et à la diffusion de documents scientifiques de niveau recherche, publiés ou non, émanant des établissements d'enseignement et de recherche français ou étrangers, des laboratoires publics ou privés. 


\section{From interferometry to color holography (Jean-Michel DESSE, ONERA)}

\subsection{Introduction}

Everyone has observed in nature the effects produced by the deviation of light beams (mirage effect, thermal convections, etc ..) or the result of luminous interference (colored fringes caused by a thin oil film on the ground). The visualizing methods of these optical phenomena are mainly based on shadowgraph, schlieren technique (chapter 7), interferometry and holography. The first one visualizes the second derivative of the refractive index, the second one shows the first derivative of the refractive index and the last one (and also under certain conditions, interferometry) allows for measuring the refractive index itself. The principle of these methods and the general information on luminous interferences are widely detailed and related by [1],[2],[3] with many and varied applications in fluid mechanics [4],[5].

This chapter presents interferometry and holography for quantitative measurements of refraction index and, consequently, density.

\subsubsection{Luminous interference}

Firstly, the interferences between luminous waves can be observed if the following conditions are met:

- They come from the same source point;

- They have the same frequency;

- The vibration directions of their luminous vectors are parallel to each other (or, at least, the components which interfere are parallel);

The first two conditions correspond to the notions of spatial coherence (the source must be seen in a very small angle) and temporal coherence (the optical path difference of the 
interfering waves must be small compared to the length of the wave packets). The last condition is related to the polarization. When two or more waves overlap, their amplitudes add to give a new wave whose amplitude depends on the phase between these waves. This phenomenon is referred as interference. A wave $U$ of amplitude $A$, of frequency $\omega$ and phase $\varphi$ can be mathematically represented by the following complex form:

$$
U=A \exp ^{i(\omega t-\varphi)}
$$

Consider two waves $U_{1}$ et $U_{2}$ (amplitude $A_{1}$ and $A_{2}$, phase $\varphi_{1}$ and $\varphi_{2}$ respectively), the wave $U=U_{1}+U_{2}$ resulting from their interference is expressed by:

$$
\begin{aligned}
& U=A_{1} \exp ^{i\left(\omega t-\varphi_{1}\right)}+A_{2} \exp ^{i\left(\omega t-\varphi_{2}\right)} \\
& =\left[A_{1}+A_{2} \exp ^{i\left(\varphi_{1}-\varphi_{2}\right)}\right] \exp ^{i \omega t} \cdot \exp ^{-i \varphi_{1}}
\end{aligned}
$$

The term $\exp ^{i \omega t}$ which represents the variation over time of luminous quantity can be set as factor and omitted from all the following calculations without inconvenience. The sensors used in optics are only sensitive to the luminous intensity, i.e. the mean value over time of the product of the amplitude of $U$ by the complex conjugate quantity $U^{*}$ :

$$
\begin{aligned}
& I=U \cdot U^{*}=\left[A_{1}+A_{2} \exp ^{i\left(\varphi_{1}-\varphi_{2}\right)}\right]\left[A_{1}+A_{2} \exp ^{-i\left(\varphi_{1}-\varphi_{2}\right)}\right] \\
& =A_{1}^{2}+A_{2}^{2}+2 A_{1} A_{2} \cos \left(\varphi_{1}-\varphi_{2}\right)
\end{aligned}
$$

The term $2 A_{1} A_{2} \cos \left(\varphi_{1^{-}} \varphi_{2}\right)$ is the interference term.

\subsubsection{Polarization}

Most light sources emit waves whose luminous vector has a quickly and randomly variable orientation (between two consecutive wave packets). This light then presents a rotational symmetry around its propagation direction. It is called natural or non-polarized. Some lasers emit a light whose luminous vector keeps a fixed orientation in space; in this case the light is said to be polarized linearly or, more briefly, polarized. Note already that it is possible 
to filter the natural light to extracting the linearly polarized light. It is also possible, always by filtering the natural light, to achieve a light whose orientation vector varies according to a simple law. Practically, the mostly used one is the elliptical polarization, where the luminous vector describes an ellipse in the wave plane, with its particular case, the circular polarization when the ellipse is reduced to a circle. Figure 8.1 gives a representation in space of these three types of polarization in which the luminous vector describes respectively a planar sinusoid, an elliptical and a circular spiral, whose pitch is equal to the wavelength of vibration in the medium considered. Finally, note that the light can be partially polarized and then considered as a mixture of natural light and polarized light. Its polarization degree is given by the ratio of polarized luminous intensity to total luminous intensity.

Figure 8.1 Different state of polarization of luminous wave.

\subsubsection{Reflection of polarized light}

Let a luminous wave reflecting on a plane diopter between two dielectric media (Figure 8.2). Each vibration of the incident light can be decomposed on two axes, the first one perpendicular to the plane of incidence, the other one located in the plane, both being perpendicular to the direction of the light propagation. It is the same for the reflected light and refracted light. 


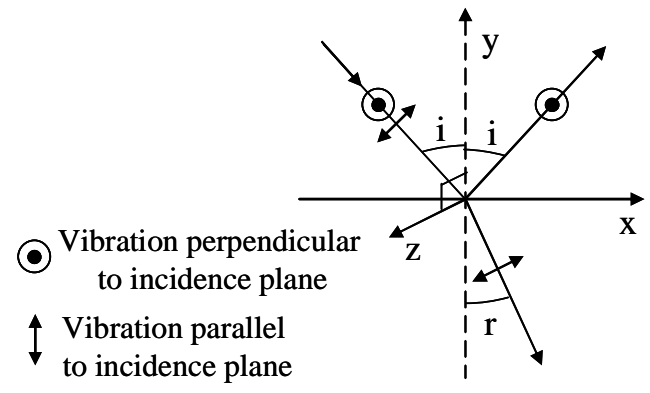

Figure 8.2 Polarization by reflection.

From the Maxwell laws, it is possible to determine the component intensities of the reflected light (Fresnel's formulas):

- For the vibration in the incidence plane $(\mathrm{x}, \mathrm{y})$ :

$$
I_{x y}=I_{0} \frac{\tan ^{2}(i-r)}{\tan ^{2}(i+r)}
$$

- For vibration in the $\mathrm{z}$ direction perpendicular to this plane:

$$
I_{z}=I_{0} \frac{\sin ^{2}(i-r)}{\sin ^{2}(i+r)}
$$

where $i$ and $r$ are the incidence and the refractive angle, respectively.

One immediately notes that $I_{z}$ can never be cancelled, while $I_{x y}$ cancels when $r=i+\pi / 2$. This particular value is called "Brewster incidence" and, at this incidence, the reflected light is completely linearly polarized, its vibration direction being normal to the incidence plane. This method is effective, but it has the disadvantage of being weakly luminous, and additionally of deflecting the light beam. 


\subsubsection{Diffraction by acousto-optic effect}

An acousto-optic modulator (AOM), also called Bragg cell, uses the acousto-optic effect to diffract and shift the frequency of light using sound waves (usually at a frequency of the order of some MHz). A piezoelectric transducer is attached to a material such as glass (e.g. Bismuth Telluride). An oscillating electric signal drives the transducer to vibrate, thus creating sound waves in the glass. These can be thought of as moving periodic planes of expansion and compression that change the index of refraction. Incoming light scatters off the resulting periodic index modulation and interference occurs. A diffracted beam emerges at an angle $\theta$ that depends on the wavelength of the light $\lambda$ relative to the wavelength of the sound $\Lambda$ in the solid following the relationship:

$$
\sin (\theta)=m \lambda / 2 \Lambda
$$

where $m=\ldots-2,-1,0,1,2, \ldots$ is the order of diffraction. In thick crystals with weak modulation as shown in Figure 8.3, only phase matched orders are diffracted; this is called Bragg diffraction (only the zero and +1 orders are diffracted).

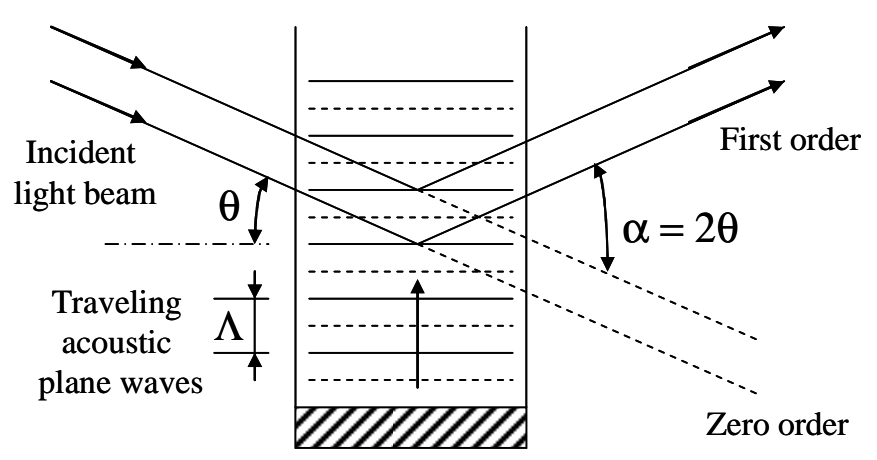

Piezoelectric transducer

Figure 8.3 Principle of acousto-optic cell. 
By simply turning the acoustic energy source on and off, the acousto-optic modulator can act as a rapid light deflector. The switching of the incident light beam to the first order diffracted beam can occur in a very short period of time $(<5 \mu \mathrm{s})$ depending only on how rapidly the acoustic wave field can be turned on and off in the volume of the flint glass traversed by the laser beam.

\subsection{Generation of luminous interferences}

Briefly, in order to obtain luminous interferences, it is necessary to form two different beams coming originally from the same light source and the beams shift has to be realized by an interferential device. There are two ways to separate the beams:

- By division of wavefront: the initial beam is split into two after passing through two small holes separated by a distance $b$, leading to the formation of Young's fringes (Figure 8.4a),

- By division of amplitude (the initial beam is split into several beams through successive reflections or transmissions (Fresnel mirror Figure 8.4b, Mach-Zehnder or Michelson interferometers).

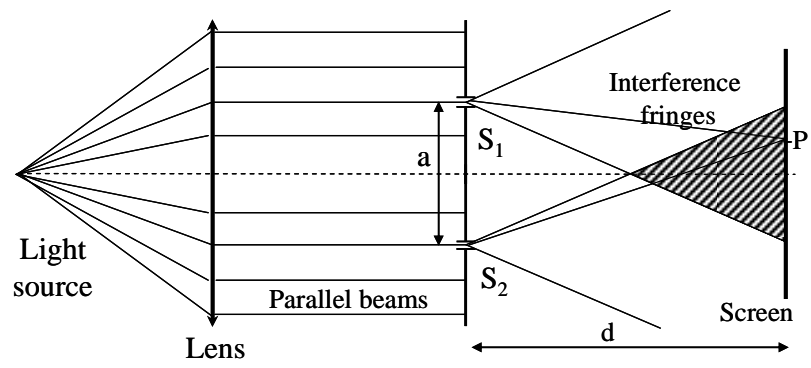

a) Division of wavefront

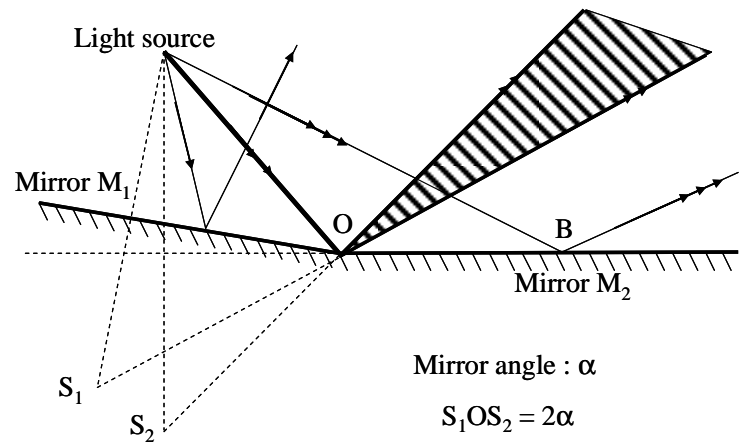

b) Division of amplitude 
Figure 8.4 Two examples of formation of interference fringes.

In Figure 8.4a, the holes can be replaced by fences and the same experiment can be realized without the convergent lens if the light source is placed far enough. Interference fringes are localized between the two diffracted beams by $S_{l}$ and $S_{2}$ separated by a distance $a$. Let $d_{l}=$ $S_{1} P$ and $d_{2}=S_{2} P$, the waves emitted by the sources $S_{1}$ and $S_{2}$ can be expressed by:

$$
U_{1}=\frac{A_{1}}{d_{1}} \exp ^{-i \frac{2 \pi}{\lambda} d_{1}} U_{2}=\frac{A_{2}}{d_{2}} \exp ^{-i \frac{2 \pi}{\lambda} d_{2}}
$$

The resulting vibration $U=U_{1}+U_{2}$ at the point $P$ is:

$$
U=\left[\frac{A_{1}}{d_{1}}+\frac{A_{2}}{d_{2}} \exp ^{-i\left(\frac{2 \pi\left(d_{2}-d_{1}\right)}{\lambda}\right)}\right] \cdot \exp ^{-i \frac{2 \pi d_{1}}{\pi}}
$$

If the distance $S_{1} S_{2}$ is small compared to $d_{1}$ or $d_{2}$, we can assume that $d 1 \approx d 2 \approx d$, where:

$$
U=\frac{1}{d}\left[A_{1}+A_{2} \exp ^{-i\left(\frac{2 \pi\left(d_{2}-d_{1}\right)}{\lambda}\right)}\right] \exp ^{-i \frac{2 \pi d}{\pi}}
$$

In this form, the interference term $2 \pi\left(d_{2}-d_{1}\right) / \lambda$ appears as a measurement of the optical path difference between the two waves incoming from $S_{1}$ and $S_{2}$. Furthermore, the factor $\exp ^{(-i 2 \pi d / \lambda)}$ cancels out when the luminous intensity is calculated (as we have seen section 8.1.1 Luminous interference). On the other hand, the intensity measurements are always relative measurements, unaffected by the constant term $1 / d^{2}$. So, they can be eliminated in the expression of the amplitude $U$ :

$$
U=A_{1}+A_{2} \exp ^{\left(-i \frac{2 \pi \Delta}{\lambda}\right)}
$$

with $\Delta=d_{2}-d_{1}$ being the optical path difference. At the point $P$, the light intensity $I_{P}$ is: 


$$
I=U \cdot U^{*}=A_{1}^{2}+A_{2}^{2}+2 \cdot A_{1} A_{2} \cos \left(\frac{2 \pi \Delta}{\lambda}\right)
$$

At any point where $\Delta=k \cdot \lambda$ (with integer $k$ ), the intensity is maximal:

$$
I_{M}=\left(A_{1}+A_{2}\right)^{2}
$$

The locations of these points are called bright fringes or luminous fringes. At any point where $\Delta=(2 k+1) \lambda / 2$, the intensity is minimum:

$$
I_{m}=\left(A_{1}-A_{2}\right)^{2}
$$

The locations of these points are called black fringes and dark fringes. In particular, if $A 1=A 2=A 0, I_{M}=4 A_{0}^{2}$ and $I_{m}=0$. If the coordinates of $S_{1}$ and $S_{2}$ sources are $(\mathrm{a} / 2,0)$ and $(-$ $\mathrm{a} / 2,0)$ and $(\mathrm{x}, \mathrm{y})$ the coordinates of any point $P$, the luminous intensity is expressed by:

$$
I=A_{1}^{2}+A_{2}^{2}+2 \cdot A_{1} A_{2} \cos \left(\frac{2 \pi a x}{\lambda d}\right) \text { with } \Delta=d_{2}-d_{1}=\frac{a \cdot x}{d}
$$

and the fringe space $f r$, i.e. the distance two dark or bright fringes, is:

$$
f r=\lambda d / a
$$

In Figure $8.4 \mathrm{~b}$, the amplitude of the incident light is split by two mirrors with a very small angle ( 1 minute to a few minutes of angle). The interference fringes are produced by the reflection on the two mirrors. In general, the interference fringes can be localized if they are observed at a precise location in space or unlocalized if they are observed in the space area where the beams overlap.

Finally, when the two beams of the interferometer are entirely separated and when one of the two beams crosses through the test section, the interferometer is of conventional type and it is called "separated beams interferometer". With this type of apparatus, the optical thickness $E$ can be directly measured in the test section. The optical thickness determines the phase delay of the 
light passing through a medium with index of refraction $n$, and it is also referred as optical path length. If the thickness $e$ of the test section is known, $E=(n-1)$.e and the following GladstoneDale relationship (reported in chapter 7 and here recalled) relates $\rho$ to $n$ :

$$
\frac{n-1}{\rho / \rho_{s}}=K
$$

in which $\rho_{s}$ is a reference value of the gas density and $K$ Gladstone-Dale constant depending on the gas. Under standard conditions $\left(0^{\circ} \mathrm{C}, 1 \mathrm{~atm}\right), \rho_{s}=1.2928 \mathrm{~kg} / \mathrm{m}^{3}$ and $K=$ $293.10^{-6}$ for dry air.

When the two interfering beams are very weakly separated, they both pass through the test section and the derivative of the optical thickness is measured along the shift direction of the two beams. This second type of interferometer is called "differential".

\subsection{Different types of interferometers}

\subsubsection{Separated beams interferometry}

\subsubsection{Michelson interferometer}

Among the most used interferometers with separated beams it is worth mentioning the Michelson interferometer [2]. A beamsplitter plate called "separating plate" divides a beam of parallel rays into two approximately perpendicular beams (Figure 8.5). These beams are reflected by plane mirrors on this separating plate and observed beyond it. They have equal luminous intensities, but as one of the beams passes twice through the separating plate while the other one is reflected twice by the separating plate, a "compensating plate" is inserted in the optical setup. The thickness of the compensating plate is equal to the thickness of the separating plate in order to compensate for the different optical lengths. The observed interference fringes are similar to 
those obtained by reflexion on the two faces of transparent plates [6]. The source being assumed extended, rings localized at infinity are observed if the two mirrors are rigorously perpendicular. On the screen, a unique black fringe is obtained because the reflexions produced by the two beams on the beamsplitter plate generate a shift of a half period for one of the beams. If one of the two flat mirrors is rotated by a small angle, straight equidistant interference fringes appear and they are localized near the flat mirror located on the optical axis of the screen and the objective. In the wind tunnel, the flat mirror is located just behind the test section where the interference fringes are localized. Two plates compensating the test section windows are inserted in the other optical arm. The measurement of the optical thickness of the gas in the test section is obtained comparing two interferograms: the first one recorded without the flow and the second one with the flow.

Figure 8.5 Michelson interferometer. 


\subsubsection{Mach Zehnder interferometer}

The Mach-Zehnder interferometer is another kind of separated beams interferometer, with the advantage of giving interference fringes which can be controlled moving the optical pieces. The optical setup presented in Figure 8.6 uses two semi-transparent plates and two flat mirrors sensibly parallel and located at the four corners of a rectangle. A beam of parallel light is splitted by a semi-transparent plate into two beams with the same intensity. One is deflected with an angle of $90^{\circ}$, while the other one proceeds with unchanged direction The second mirror returns also at $90^{\circ}$ the beam which has crossed the beamsplitter plate. The second beamsplitter gathers the two beams to make them interfering. It can be shown that the plane in which the interference fringes are located is parallel to the bisector of the two large sides of the rectangle and passes by the intersection point $\mathrm{O}$ of this axis. The interference fringes can be observed through an objective which is focused in the plane normal to the beam and containing the point O.

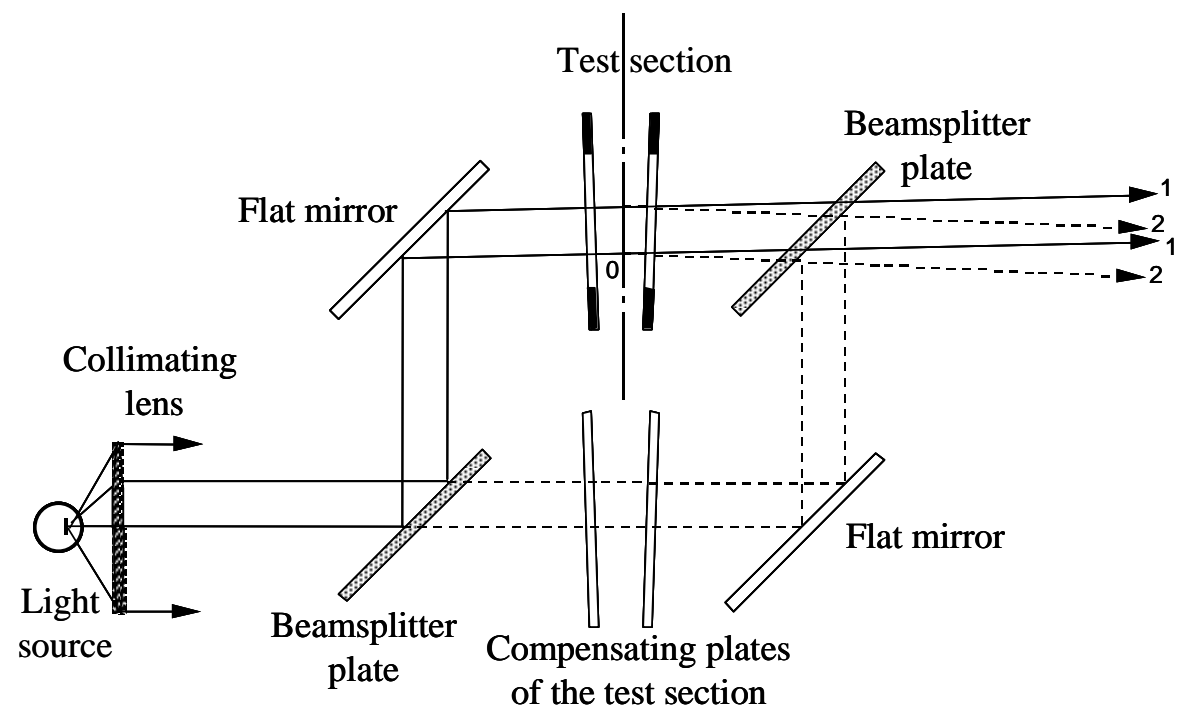

Figure 8.6 Mach-Zehnder interferometer. 
The test section is crossed by one of the beams and the test section windows are compensated by two identical plates located in the path of the other beam. Mirrors and plates are adjusted in order to locate the plane of the fringes in the middle of the test section. By translating a mirror or a plate, the central fringe is brought into the test section. The optical setup can be adjusted with a uniform background fringe or with straight fringes oriented in the most suitable direction. An example is given in Figure 8.7 where the interaction between a shock wave and the boundary layer on the upper and the lower floors of test section is visualized. On interferogram a) recorded with narrow fringes, one can follow the fringes step through the shock wave and the analysis can be conducted on all the interferogram. On interferogram b) recorded with a uniform background tint, it is not possible to follow the fringes through the shock wave, but a small variation in the optical thickness is observed between two successive reflections [7]. This example shows that the interferometer has to be correctly adjusted before recording to analyze strong or weak variations in the refractive index. A quantitative analysis of interferograms requires the identification of the fringes and of their shift across flow discontinuities.
a) narrowed fringes interferogram
b) uniform background interferogram

Figure 8.7 Interaction between a shock wave and the wind tunnel floors. 


\subsubsection{Differential interferometry}

When the density gradients are relatively weak, the differential interferometry of polarized white light is more suited. It produces coloured interferograms, the analysis of which yields the density field after calibration of the whole setup [8],[9]. The principles of differential interferometry have been discussed in details in [10]. A birefringent crystal (Wollaston biprism, for example), constituted by two quartz or calcite prisms, crossed and bonded with an angle $\alpha$ (Figure 8.8a), decomposes a polarized light vibration in a given direction into two orthogonal coherent vibrations of approximately equal amplitudes. With the type of crystal used, the birefringence is described as uniaxial, meaning that there is a single direction governing the optical anisotropy whereas all directions perpendicular to it are optically equivalent. This special direction is known as the optic axis of the material. Light whose polarization is perpendicular to the optic axis is governed by a refractive index $n_{\mathrm{o}}$ (for "ordinary"). Light whose polarization is in the direction of the optic axis sees an optical index $n_{\mathrm{e}}$ (for "extraordinary"). For any ray direction there will be a polarization direction perpendicular to the optic axis, and this is called an ordinary ray. However, for most ray directions the other polarization direction will be partly in the direction of the optic axis, and this is called an extraordinary ray. The ordinary ray will always experience a refractive index of $n_{0}$, whereas the refractive index of the extraordinary ray will be in between $n_{\mathrm{o}}$ and $n_{\mathrm{e}}$, depending on the ray direction as described by the index ellipsoid. Here, one of the prism has its optical axis perpendicular to its edge. Each incident ray is polarized at $45^{\circ}$ with respect to the quartz axis so that, after crossing the biprism, the emerging rays are separated by a small birefringence angle $\varepsilon=\varepsilon(\lambda)$. If $n_{e}$ and $n_{o}$ are respectively the extraordinary and ordinary refractive indices of the crystal, the birefringence angle is given by: 


$$
\varepsilon=2\left(n_{e}-n_{o}\right) \tan \alpha
$$

As a consequence, two rays cross the phase object at slightly different locations. After passing through the phase object the two rays can interfere in an analyser. The difference in optical path, which is put into evidence in this way, is characteristic of the body under observation since the setup is autocompensated. Eq. (8.17) shows that the sensitivity of the measurement depends on the type of birefringence crystal used and also on the bonding angle. For instance, if high sensitivity is required, calcite material will be preferred with respect to quartz because the calcite birefringence is twenty times higher than the quartz birefringence.

Very often, large objects have to be analyzed (more than $100 \mathrm{~mm}$ in diameter) so that a diverging light beam crosses the Wollaston prism and then, it is sent towards the object under analysis. If a strictly uniform background color is required (no phase shift), the Wollaston prism should be crossed by a parallel beam. If it is not the case, one could expect to have continuously varying colours in the field of observation. To reasonably compensate for this defect [11] and to obtain large field birefringent compensator (Figure 8.8b) it is appropriate to add $\lambda / 2$ plates before and past the bonded prisms and two plane crystals after and before the system.

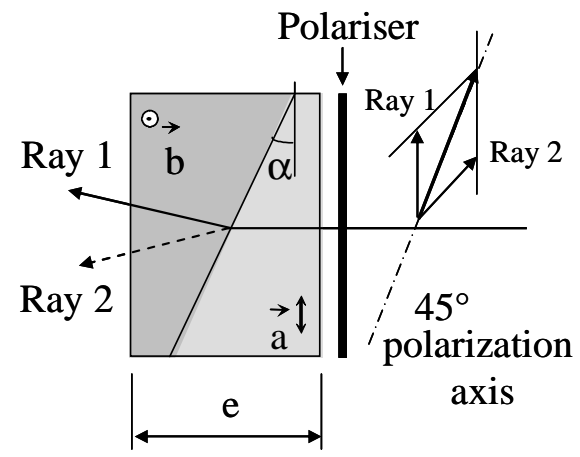

a) Standard Wollaston biprism

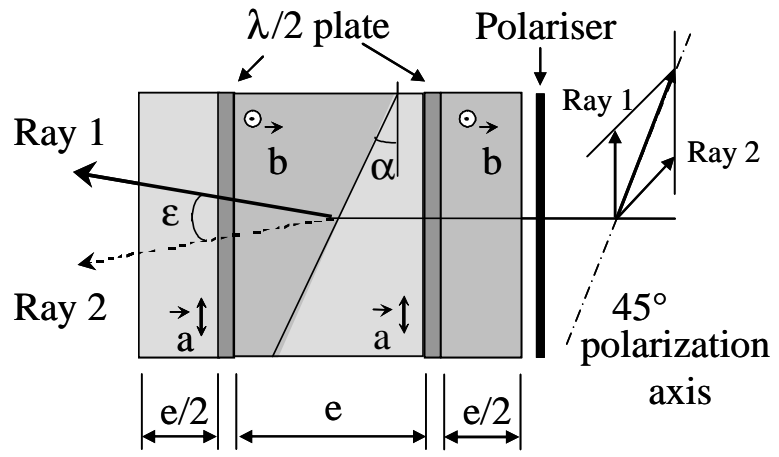

b) Large field Wollaston biprism

Figure 8.8 Modification of Wollaston biprism for analyzing very large field. 
In this way, some authors performed experiments where the setup includes a Wollaston prism which highly separates the interfering beams. This type of setup is equivalent to conventional reference beam interferometry as the interferograms can be interpreted directly, but remains differential since one of the beams - the reference one - lies inside the undisturbed upstream flow [12],[13],[14],[15].

Several optical setups can be designed to include one or two Wollaston prisms. Here two possibilities are considered:

- the first one includes one Wollaston prism. The optical beams cross the test section twice, thus making the apparatus compact and doubling its sensitivity. Adjustment is quick and easy as no compensation is needed for the optical thickness of the test section windows since both beams pass through them. In the differential interferometer of Figure 8.9, the prism is placed at right angle from the axis of the spherical mirror, close to its centre of curvature (the prism is said to be centred). Before passing through the prism, the beam goes through a polarizer; on the return leg, after leaving the prism, the beams pass through an analyser which is either parallel or orthogonal to the polarizer. As the optical beams pass through the prism twice, the initial path difference can be compensated by translating the prism. The system is also self-compensating as, the prism being fixed, any deviation in the light beams generates variations of the optical thickness in the air and in the prism which cancel: the observed path difference is thus directly related to the properties of the observed medium. If a white light is used (high pressure xenon source, for example), a system of colored fringes is observed, the tints of which are arranged in series roughly equivalent to the Newton scale [16]. The observation of the interference fringes yields a measurement of the optical path difference which exists between the 
beams after the crossing of the crystal, the ambient air and the test section: from that, one infers the component of the gas density gradient along the direction perpendicular to the fringes. Then, the gas density itself is obtained by integration.

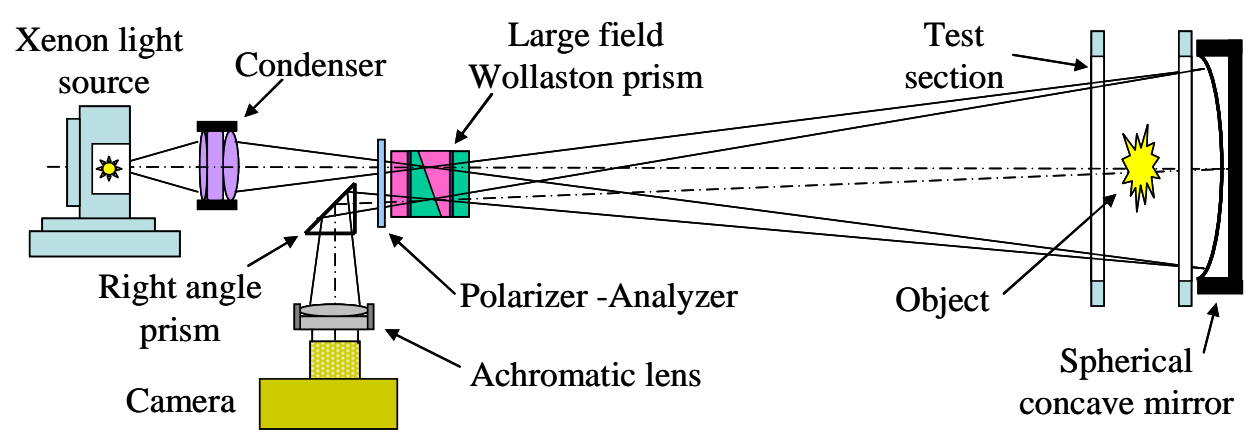

Figure 8.9 Differential interferometer with double crossing and one Wollaston prism.

- for the second one, the optical setup of Figure 8.9 is changed from a double crossing diverging beam to a single parallel beam setup as shown in Figure 8.10. It requires two Wollaston prisms and two achromatic "Clairaut" lenses (designed to minimize chromatic and spherical aberrations), one on each side of the test section. The two Wollaston prisms, mounted “upside down”, are located at each focusing point. A linear polarizer is used to adjust the input polarization and an analyser is than placed behind the second prism in order to produce interferences between the orthogonally polarized beams [20].

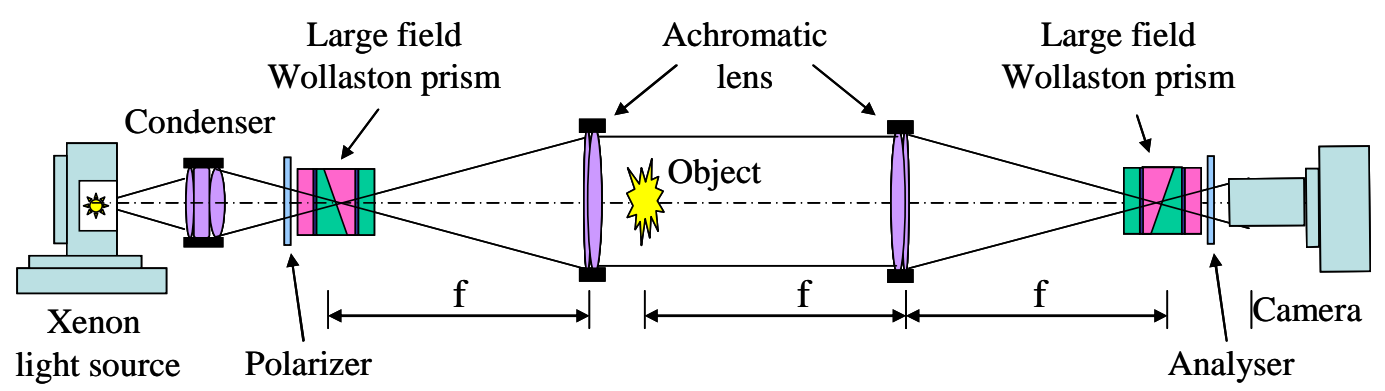

Figure 8.10 In line differential interferometer with single crossing and two Wollaston prisms. 
An alternative of this setup can be used when very large fields of analysis have to be analyzed. Due to the very high cost of the achromatic "Clairaut" lenses, a "Z" optical setup is preferred where two spherical mirrors replace the two achromatic lenses. In order to avoid astigmatic aberrations, the angle of opening has to be less than $10^{\circ}$ (Figure 8.11).

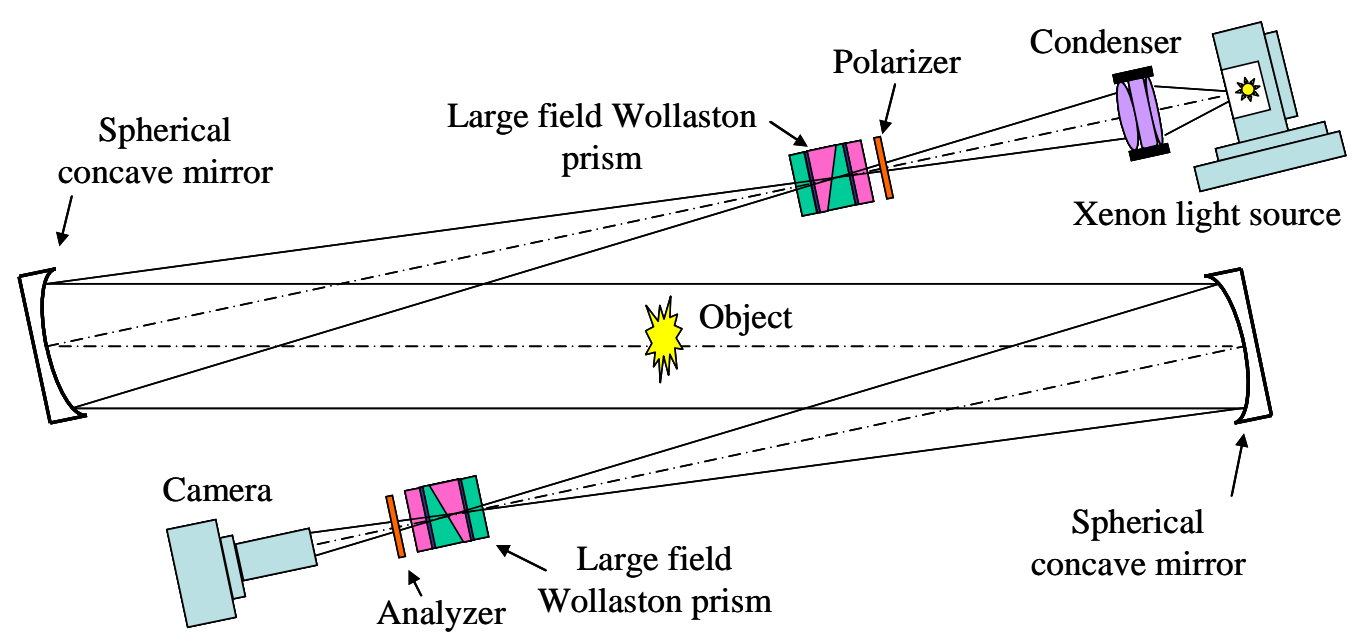

Figure 8.11 "Z" optical setup with single crossing and two Wollaston prisms

\subsubsection{Interferograms analysis}

\subsubsection{Setup calibration for a manual analysis (2D flows)}

The manual analysis of interferograms can be made if the calibration of the position $\xi$ of the Wollaston prism versus the observed colours in the interferometer has been realized before. $\xi$ is the Wollaston prism displacement measured in the plane perpendicular to the optical axis along $x$ or $y$. The light deviation $\theta_{x}$ or $\theta_{y}$ is obtained from the following reasoning. Let $R$ be the radius of curvature of the spherical mirror located behind the test section (Figure 8.9) or $f$, the focal length of the achromatic lens or the spherical mirror (Figure 8.10) and $L^{\prime}$ the virtual distance between the middle of the test section and the spherical mirror, the distance $d x$ or $d y$ between the two interfering rays in the test section is equal to: 


$$
d x=\varepsilon\left(R-L^{\prime}\right) \text { or } d x=\varepsilon\left(f-L^{\prime}\right)
$$

It is easily shown that, under no flow conditions, the relative displacement $\xi-\xi_{o}$ of the prism induces a path difference $\Delta$ between the outgoing and returning rays in the prism which is:

$$
\Delta=2 \varepsilon\left(\xi-\xi_{0}\right)
$$

The result of this path difference can be observed in the interferometer as a uniform variation in the background colour (Figure 8.12). Here, as the polarizer and the analyser are parallel, a white fringe is obtain for $\xi-\xi_{0}=0$.

Figure 8.12 Prism calibration - variation of colors with $\left(\xi-\xi_{0}\right)_{\mathrm{mm}} \times 10^{2}$.

When a flow exists in the test section, a light ray crossing the medium under analysis is deviated by an angle $\theta_{x}$ or $\theta_{y}$ equal to:

$$
\theta_{x}=d E / d x \text { or } \theta_{y}=d E / d y
$$

where $d E$ is the difference in optical thickness of the medium for the two interfering rays. As the test section is crossed twice by the light rays, the path difference $\delta$ produced by the observed medium is: 


$$
\delta=2 d E
$$

From the relationships (8.18) and $\theta_{x}=d E / d x$ or $\theta_{y}=d E / d y \quad(8.2(8.20)$, equation

(8.21) becomes:

$$
\delta=2 \varepsilon\left(R-L^{\prime}\right) \theta_{x}
$$

When the tints observed under flow and no flow conditions are identical, the path difference $\delta$ and $\Delta$ are equal, yielding:

$$
\delta=2 \varepsilon\left(R-L^{\prime}\right) \theta_{x}=2 \varepsilon\left(\xi-\xi_{0}\right)
$$

The light deviation is then expressed as:

$$
\theta_{x}=\left(\xi-\xi_{0}\right) /\left(R-L^{\prime}\right)
$$

The light deviation is obtained from the measurement of only two lengths: the prism displacement $\xi-\xi_{o}$ and the radius of curvature of the spherical mirror. Progressively moving the prism and noting the colours in the interferometer are sufficient to obtain the calibration curve of the optical setup.

In the analysis of the interferograms, one starts from a position where the background colour is uniform and where the gas density is known and, by moving normally to the interference fringes, say $x$, one notes the position of the observed tints. The calibration curve obtained from equation $\theta_{x}=\left(\xi-\xi_{0}\right) /\left(R-L^{\prime}\right) \quad$ (8.2(8.24) yields the light deviation at every point [9]. The optical thickness $E$ is obtained by integrating the light deviation: 


$$
E-E_{0}=\int \theta_{x} \cdot d x
$$

If the gas density is known at one point in the field, the Gladstone-Dale relationship (Eq.8.16) allows to obtain the refractive index at this point. For a two-dimensional flow and if $e$ is the test section width, the optical thickness is given by:

$$
E_{0}=\left(n_{0}-1\right) . e=K . e \cdot \frac{\rho_{0}}{\rho_{s}}
$$

As the optical thickness is proportional to the gas density, one obtains:

$$
\left(E-E_{0}\right) / E_{0}=\left(\rho-\rho_{0}\right) / \rho_{0}
$$

from which:

$$
\rho / \rho_{0}=1+\left(E-E_{0}\right) / E_{0}
$$

\subsubsection{Case of axisymmetric flow}

The analysis of axisymmetric flow implies an additional complexity. If the interferogram is recorded with horizontal fringes, the analysis has to be realized along parallels to $\mathrm{Oz}$ as shown in Figure 8.13.

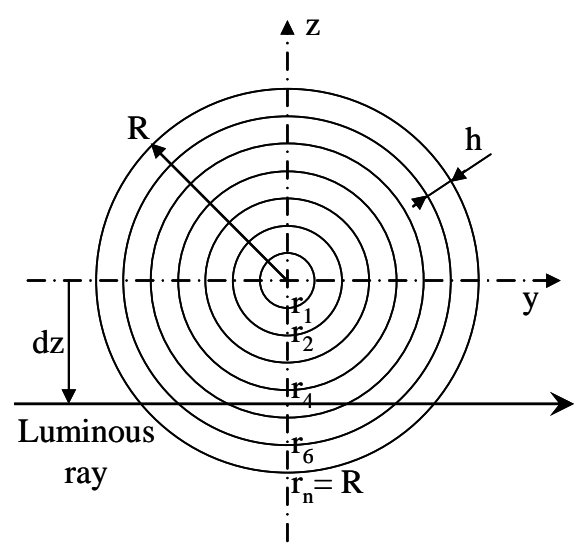

Figure 8.13 Division of asymmetric field in $N$ rings of the same thickness $h$.

Between two horizontal lines $y_{0}$ and $y$, the deviation of luminous ray is given by: 


$$
\theta_{z}=\int_{y_{0}}^{y} \frac{1}{n} \frac{d n}{d z} d y=\int_{y_{0}}^{y} \frac{d \bar{n}}{d z} d y
$$

with $\bar{n}=\log n$. In cylindrical coordinates, the deviation $\theta_{z}$ crossing an axisymmetric flow at abscissa $\mathrm{z}$ is given by [1]:

$$
\frac{\theta_{z}}{2 z}=G(z)=\int_{z}^{R} f(r) \frac{2 r d r}{\sqrt{r^{2}-z^{2}}}
$$

where $\mathrm{R}$ is the radius of the optically homogeneous medium and $r=\sqrt{y^{2}+z^{2}}$. The function:

$$
f(r)=\frac{\partial(\log n)}{\partial\left(r^{2}\right)}=\frac{\partial \bar{n}}{\partial\left(r^{2}\right)}
$$

is the unknown to be determined, where $n$ is the refraction index of the gas. Equation (8.30) is solved assuming the flow to be axisymmetric. The radius $R$ of the inhomogeneous medium is divided into $N$ rings of the same thickness $h$. With $r_{i}=i h$, the experimental values $G_{i}=G\left(z=r_{i}\right)$ are known. Then, the continuous variations of the refractive index are replaced by a sequence of discontinuities separating constant values in each ring. We write: $f_{i}=f(r)$ for $r_{i-1}<r<r_{i}$. Equation $\frac{\theta_{z}}{2 z}=G(z)=\int_{z}^{R} f(r) \frac{2 r d r}{\sqrt{r^{2}-z^{2}}}$ using the method suggested in [1]. The system is solved recursively: knowing the values of $f_{i}$ for $k+1 \leq i \leq N$, we can deduce the values of $f_{k}$. The external flow refractive index $n_{e}$ is determined from the density measurement $\rho_{e}$ and the Gladstone-Dale equation. The density in each ring is given by: 


$$
\frac{\rho}{\rho_{e}}=\frac{n-1}{n_{e}-1}
$$

\subsubsection{Interference fringes modelling}

In the case of the recording and the automatic processing of interferograms, the approach is quite different because it is based on the spectral characterization of the whole interferometric setup, the aim being to recreate the scale of experimental colours of the interferometer on a computer. For that, we will show that it is enough to analyze the spectrum of the light source and to take into account the transfer functions of the interferometer optical components and of the three red (R), green $(\mathrm{G})$ and blue (B) filters of the camera used to digitize the interferograms. Several configurations can be met in the recording or restitution of the interferograms. In the recording, an interferogram can be either directly digitized, or recorded on a film and digitized later. In the processing, the digitization procedure of the film requires that the interferogram is illuminated with the same light source as used in the recording even if, in some cases, the light sources are different, especially when a spark source is used for the recording, while the restitution is always made with a continuous light source.

To build the colors of interference fringes, it is necessary to know the spectrum of the light source, the transfer function of the internal optical components and also of the three filters of the video camera. An example is shown in Figure 8.14. The spectrum of a XBO 150 Watt Xenon light source has been recorded through the interferometer when the Wollaston prism is precisely located at the centre of curvature of the spherical mirror and under no-flow conditions. In this manner, there is no optical path difference and only the effect of the internal optical components of the interferometer is taken into account. The spectral analysis of the light source is performed using a monochromator and a photomultiplier. The bell-shaped curves represent the spectral attenuation functions of the three internal filters of the video camera used either to 
directly record the interferogram from the interferometer, or to digitize interferograms recorded on film. The video camera considered here is a SONY 325P having separated RGB outputs for which the three filters transfer functions $\operatorname{Fr}(\lambda), \operatorname{Fg}(\lambda)$ et $\operatorname{Fb}(\lambda)$ have been given by the manufacturer.

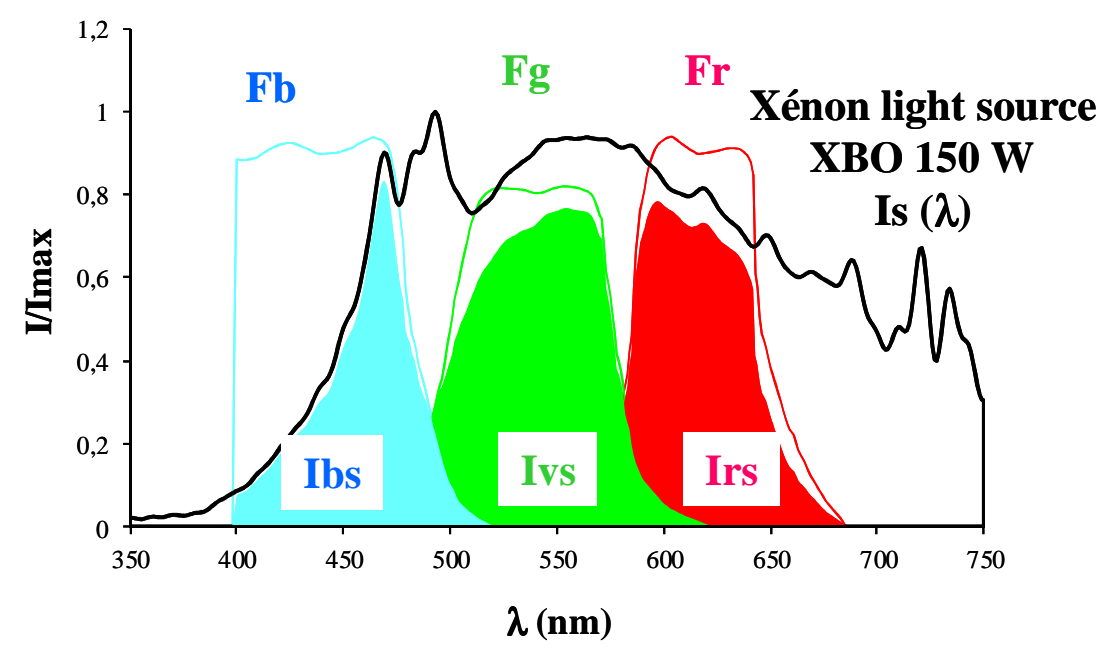

Figure 8.14 Spectra of xenon light source and cameras filters.

In Figure 8.14 Spectra of xenon light source and cameras filters., the spectral intensity $I_{S}(\lambda)$ of the light source is superimposed on the three bell curves and the spectral intensities $I_{R S}(\lambda), I_{G S}(\lambda)$ et $I_{B S}(\lambda)$ are computed and drawn through the three filters with the following relations:

$$
\begin{aligned}
& I_{R S}=I_{s}(\lambda) \cdot F_{r}(\lambda) \\
& I_{G S}=I_{s}(\lambda) \cdot F_{g}(\lambda) \\
& I_{B S}=I_{s}(\lambda) \cdot F_{b}(\lambda)
\end{aligned}
$$

This amounts to illuminating the test section with three light sources, the spectra of which are known and shifted in the wavelength scale. In monochromatic light of wavelength $\lambda_{0}$, the luminous intensity of the interference fringes is expressed by: 


$$
I(\Delta)=4 I_{0} \cos ^{2}\left(\pi \Delta / \lambda_{0}\right)
$$

if the interfering vibrations have the same amplitude. In this precise case, the path difference $\Delta$ does not depend on the light wavelength $\lambda_{0}$, and, with the relation $\Delta=2 \varepsilon\left(\xi-\xi_{0}\right)$

(8.(8.19), the intensity distribution of the fringes is given by:

$$
I(\xi)=4 I_{0} \cos ^{2}\left(2 \varepsilon\left(\lambda_{0}\right)\left(\xi-\xi_{0}\right) / \lambda\right)
$$

Relationship $I(\xi)=4 I_{0} \cos ^{2}\left(2 \varepsilon\left(\lambda_{0}\right)\left(\xi-\xi_{0}\right) / \lambda\right) \quad$ (8.(8.35) can be extended to the interferences in white light. The constant luminous intensity $I_{0}$ is substituted by $I_{S}(\lambda)$ and the birefringence angle becomes a function of the wavelength. The resultant intensity is then computed as the following integral:

$$
I(\xi)=4 . \int_{\lambda=0.4 \mu m}^{\lambda=0.8 \mu m} I_{s}(\lambda) \cos ^{2}\left(2 \varepsilon(\lambda) \cdot\left(\xi-\xi_{0}\right) / \lambda\right) \cdot d \lambda
$$

$\varepsilon$ varies little with wavelength and to each $\xi$ can be associated some mean path difference $\Delta$ using the value of $\varepsilon$ for a given reference wavelength $\lambda_{0}\left(\lambda_{0}=560 \mathrm{~nm}\right)$.

$$
\begin{gathered}
\Delta=2 \varepsilon\left(\lambda_{0}\right) \cdot\left(\xi-\xi_{0}\right) \\
I(\Delta)=4 \cdot \int_{\lambda=0.4}^{\lambda=0.8} I_{s}(\lambda) \cos ^{2}\left(\frac{\pi \Delta \varepsilon(\lambda)}{\varepsilon\left(\lambda_{0}\right)}\right) \cdot d \lambda
\end{gathered}
$$

To calculate the intensity of the interference fringes on the red, green and blue channels, the relation $I(\Delta)=4 . \int_{\lambda=0.4}^{\lambda=0.8} I_{s}(\lambda) \cos ^{2}\left(\frac{\pi \Delta \varepsilon(\lambda)}{\varepsilon\left(\lambda_{0}\right)}\right) \cdot d \lambda \quad$ (8.(8.38) is applied using the three sources

$$
\begin{aligned}
I_{R S} & =I_{s}(\lambda) \cdot F_{r}(\lambda) \\
\text { computed from } I_{G S} & =I_{s}(\lambda) \cdot F_{g}(\lambda) \quad(8 .(8.33): \\
I_{B S} & =I_{s}(\lambda) \cdot F_{b}(\lambda)
\end{aligned}
$$




$$
\begin{aligned}
& I_{R}(\Delta)=4 \cdot \int I_{R S}(\lambda) \cdot \cos ^{2}\left(\pi \Delta \varepsilon(\lambda) /\left(\lambda \cdot \varepsilon\left(\lambda_{0}\right)\right)\right) \cdot d \lambda \\
& I_{G}(\Delta)=4 \cdot \int I_{G S}(\lambda) \cdot \cos ^{2}\left(\pi \Delta \varepsilon(\lambda) /\left(\lambda \cdot \varepsilon\left(\lambda_{0}\right)\right)\right) \cdot d \lambda \\
& I_{B}(\Delta)=4 \cdot \int I_{B S}(\lambda) \cdot \cos ^{2}\left(\pi \Delta \varepsilon(\lambda) /\left(\lambda \cdot \varepsilon\left(\lambda_{0}\right)\right)\right) \cdot d \lambda
\end{aligned}
$$

The results are sent to the three RGB inputs of an image processing board and the image is obtained by superposition of the three RGB planes. The resulting total intensity will be:

$$
I_{T}(\Delta)=I_{R}(\Delta)+I_{G}(\Delta)+I_{B}(\Delta)
$$

To correctly compare experimental and theoretical fringes, the minimum and maximum limits of the experimental fringes have to be computed in order to have the three sources obtained from equation (8.39) fit within these limits. The white central fringe intensity will give the maximum values $R_{\max }, G_{\max }$ and $B_{\max }$, while the minimum values $R_{\min }, G_{\min }$ and $B_{\min }$ are computed from the darker first order tint. Therefore, the signal sent, for example, to the red input is:

$$
\begin{aligned}
& I_{m r}(\delta)=R_{\text {min }}+\left(R_{\max }-R_{\min }\right) \cdot I_{R}(\delta) /\left|I_{R}(\delta)\right| \\
& I_{m g}(\delta)=G_{\text {min }}+\left(G_{\max }-G_{\min }\right) \cdot I_{G}(\delta) /\left|I_{G}(\delta)\right| \\
& I_{m b}(\delta)=B_{\text {min }}+\left(B_{\text {max }}-B_{\text {min }}\right) \cdot I_{B}(\delta) /\left|I_{B}(\delta)\right| \\
&\left|I_{R}(\delta)\right|=\max \left(I_{R}(\delta)\right), \\
& \text { where }\left|I_{G}(\delta)\right|=\max \left(I_{G}(\delta)\right), \\
&\left|I_{B}(\delta)\right|=\max \left(I_{B}(\delta)\right)
\end{aligned}
$$

Figure 8.15 shows the comparison between experimental fringes (on the bottom) and numerical fringes (on the top): practically no differences do exist. The luminous intensity $I_{T}(\delta)$ of these fringes are drawn below. It can be seen that the luminance (visual excitation luminance intensity) and chrominance (qualitative color) are astonishingly well reproduced. The good agreement of the experimental and theoretical fringes makes it legitimate to apply the theoretical path difference scale to the experimental colors [9]. 
Figure 8.15 Comparison between the fringes colors and the luminous intensities.

Very often, the interferograms are recorded on photographic films and the film spectral response has to be taken into account. The following procedure can be assumed: under no flow conditions, the Wollaston prism is located at the centre of curvature of the spherical mirror and an interferogram is recorded with the white background color (parallel polarizer and analyzer). After development of the interferogram, it is illuminated with the same light source in order to analyze the spectrum of the uniform white tint recorded on the film. The spectral shape of the light source is attenuated by the film spectral response. In order to avoid problems induced by the film development, the uniform background interferogram and the measurement interferogram are simultaneously developed in the same baths.

The RGB transfer functions of the video camera interfere at the restitution in the interferogram digitization and the process of fringes comparison described above is applied. In the experiments, one white background interferogram is recorded on the film for determining its spectrum. Then, one other interferogram is recorded in narrow fringes to yield the minima and 
maxima values and to allow a comparison of the computed and experimental fringes. It is then no longer necessary to calibrate the interferometric system before recording the interferograms. For example, the sensitivity measurement can be evaluated for a biprism angle of $4^{\circ}$, a spherical mirror of $2500 \mathrm{~mm}$ in the focal length of radius of curvature, an optical setup with 2 crossings of the test section and a flow two-dimensional with a test section width of $42 \mathrm{~mm}$. The abrupt change between two colours perceived by the eye is located in the tints of the first order of the Newton scale and it is induced by a variation in the minimum path difference of $30 \mathrm{~nm}$. In these conditions, it is possible to measure a variation density of $5.0110^{-4} \mathrm{~kg} / \mathrm{m}^{3}$.

\subsection{Principles of three-wavelength differential interferometry}

\subsubsection{Advantage of using three wavelength source}

In monochromatic interferometry (for instance, $\lambda=647 \mathrm{~nm}$ ), it is well known that the classical interference pattern is represented by a succession of dark and bright red fringes. For two successive fringes, the optical path difference is equal to the wavelength of the laser source (Figure 8.16a). Unfortunately, the zero order of interferences fringes can never be identified and it is one of the major difficulties with interferences fringes in monochromatic light. Sometimes, it is not possible to follow the displacement of the fringes through a shock wave, for example, or to count the fringe number in a complex flow. When the light source is a continuous source (500 Watt xenon, see Figure 8.16b), the interference pattern is a coloured fringe pattern in a sequence approximately matching Newton's colour scale. This fringes diagram exhibits a unique white fringe, visualizing the zero order of interference and it allows one to measure very small path differences, because six or seven different colours define the interval 0-0.8 microns. When the path difference is greater than three or four microns, instead, the colours can no longer be 
separated and the larger path differences cannot be correctly measured [17]. Figure 8.16c shows the fringes obtained with a laser that emits three different wavelengths (one blue line, one green line and one red line). One can see that the disadvantages of the two spurious sources (Figure 8.16a,b) disappear. The zero order is always identifiable and the colours always remain distinguishable for the small and the large path differences. The interference pattern also presents the following peculiarity: while the white fringe is not visible on the interferogram, the sequence of three successive colours in the diagram is unique.

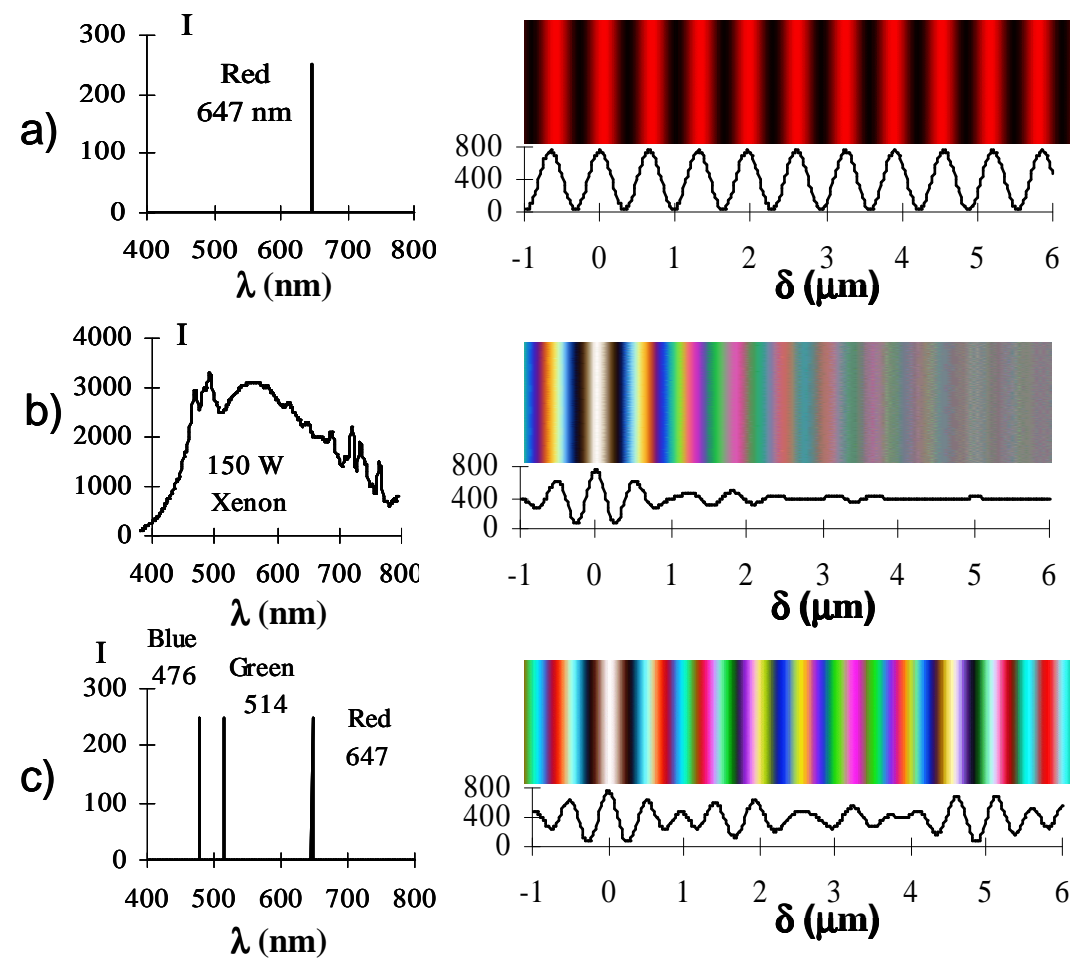

Figure 8.16 Spectra and interference fringes given by three different light sources

\subsubsection{Choice of three wavelengths}

First, we must remember that each wavelength or each color can be specified in terms of equivalent stimuli. The tristimulus values $(\mathrm{x}, \mathrm{y}, \mathrm{z})$ were adopted by the International Commission on Illumination (CIE, Commission Internationale de l'Eclairage [18]) for the various spectrum 
colors. They indicate the amount of each of the CIE primaries that is required to match the color of one watt of radiant power of the indicated wavelengths. The tristimulus system is based on visually matching a colour under standardized conditions against the three primary colours: red, green, and blue; the three results are expressed as $\mathrm{X}, \mathrm{Y}$, and Z, respectively, and are called tristimulus values. These values specify not only colour but also visually perceived reflectance, since they are calculated in such a way that the Y value equals a sample's reflectivity when visually compared with a standard white surface by a standard viewer under average daylight. The tristimulus values can also be used to determine the visually perceived dominant spectral wavelength (which is related to the hue) of a given sample. Such data can be graphically represented on a standard chromaticity diagram (Figure 8.17) based on the values $x, y$, and $z$, where $\mathrm{x}=\mathrm{X} /(\mathrm{X}+\mathrm{Y}+\mathrm{Z}), \mathrm{y}=\mathrm{Y} /(\mathrm{X}+\mathrm{Y}+\mathrm{Z})$, and $\mathrm{z}=\mathrm{Z} /(\mathrm{X}+\mathrm{Y}+\mathrm{Z})$. Note that $\mathrm{x}+\mathrm{y}+\mathrm{Z}=1$; thus, if two values are known, the third can always be calculated, so the $\mathrm{z}$ value is usually omitted. The $\mathrm{x}$ and $\mathrm{y}$ values together constitute the chromaticity of a sample. Light and dark colours that have the same chromaticity (and are therefore plotted at the same point on the two-dimensional chromaticity diagram) are distinguished by their different $\mathrm{Y}$ values (luminance, or visually perceived brightness).

When their $\mathrm{x}$ and $\mathrm{y}$ coefficients are plotted on a chromaticity diagram, the spectral colours from $400 \mathrm{~nm}$ to $700 \mathrm{~nm}$ follow a horseshoe-shaped curve; the non-spectral violet-red mixtures fall along the straight line joining the $400 \mathrm{~nm}$ point to the $700 \mathrm{~nm}$ point. All visible colours fall within the resulting closed curve, as shown in the standard chromaticity diagram. Points along the circumference correspond to saturated colours; pale unsaturated colours appear closer to the centre of the diagram. The achromatic point is the central point at $\mathrm{x}=0.33, \mathrm{y}=0.33$ (shown as a small circle in the Figure 8.17), where visually perceived white is located (as well as 
the pure greys and black, which vary only in the magnitude of the luminance Y). The line which links the wavelength from $380 \mathrm{~nm}$ to $780 \mathrm{~nm}$ is called the line of purples. For example, the dotted line triangle gives the coordinates of the fundamental colours of the RGB system (Red, Green and Blue). For television, it is the dashed line triangle which has been adopted by the NTSC standard.

In the case of three wavelength differential interferometry, the chosen wavelengths create a three-color base that can reproduce a maximum numbers of colors and the laser output power enables the interferograms to be recorded at a high framing rate as required by unsteady flows (exposure time of the order $1 \mu \mathrm{s}$ ). Three wavelengths interferometry requires, in principle the use of three different lasers. It is possible to avoid the use of three different lasers, for example, by using an ionised gas laser (mixed Argon and Krypton) that produces approximately 10 visible lines. By selecting the following wavelengths: $\lambda_{1}=476.7 \mathrm{~nm}, \lambda_{2}=514.5 \mathrm{~nm}, \lambda_{3}=647.1 \mathrm{~nm}$ of this laser, we obtained the RVB triangle whose apex are represented on Figure 8.17. 
Figure 8.17 Triangle defined by the fundamental colours of Innova spectrum laser.

All the colours which can be found in the scale of tints of the interferometer are included in this triangle which is the biggest triangle that can be created with the wavelengths generated by an Argon and Krypton laser.

\subsubsection{Contribution of color in oil-film thickness measurement}

An example is given to illustrate the color contribution to directly determining the sign of the change in the oil film thickness to be used for the measurement of wall shear stresses (see chapter 12) from the knowledge of the color in Newton's tints scale [19]. A thicker oil film corresponds to a longer optical path. Figure 8.18 shows an example: three different light sources are used to record the oil film interference fringes in the vicinity of the boundary layer transition region on a flat plate. The flow is from the left to the right and the central part of the interferogram has been isolated in order not to take into account the test section lateral boundary layers.

Figure 8.18 Interference fringes obtained with three different light sources 
Interferograms 1 and 2 show interference fringes recorded with green and red lines, respectively issued from an argon and a krypton laser. The simple visualization of dark and bright fringes does not allow the unambiguous determination of the evolution of the oil film thickness. Interferogram 3 is obtained with a xenon light source and the knowledge of Newton's tints scale allows determining the variation of the oil film thickness without any doubt. Moreover, since no color is enclosed with two identical tints in the Newton's scale, it is easy to detect the extrema of the oil film thickness profile. This has the advantage of allowing to determine easily the gradient changes of the oil film. In interferogram 3 of Figure 8.18, one can see that the pale green fringe is enclosed within two fringes having the same red color (first change in the oil film thickness slope sign) and that the yellowish fringe is enclosed within two identically purplish-red colored fringes (second change of the slope sign). Analysis of the interferogram colors indicates that the oil film thickness is increasing upstream of the location of the pale green color, then decreasing up to that of the yellowish color and increasing again downstream of it. Also note that the oil film thickness experiences a small variation in the downstream region. Better sensitivity is obtained with a white light source because several fringes are visible downstream of the central part of the model. In monochromatic light (argon or krypton lasers), it is only possible to distinguish a variation of one fringe.

\subsection{Application to flows}

\subsubsection{Application to 2D unsteady subsonic wake flows}

In this example, the unsteady wake flow of schematic turbine blades at Mach 0.4 is analysed [20]. The unsteady pressure signals are simultaneously recorded around the trailing edge in order to be able to synchronize the acquisition of images and of pressure variations. Two 
successive interferograms and the gas density field are given in Figure 8.19. The gas density $\rho$ is referenced to the upstream gas density $\rho_{\infty}$. The present model has a circular trailing edge with $D=15 \mathrm{~mm}$ and the boundary layer is quasi-laminar. Due to the shifting of the two interfering rays $(d x=1.57 \mathrm{~mm})$, the interferograms cannot be analyzed down to the model wall. The interferograms analysis is conducted using the numerical model described in section 8.3.3.3 Interference fringes modelling. For example, an analysis conducted following a vertical line is only possible until one of two interfering rays is blocked by the model. In the conditions of the optical setup, the analysis is made down to $\mathrm{dx} / \mathrm{D}=0.1$ from the model wall and the gas density at the wall is obtained through extrapolation of the data. As a vortex can be defined as rotational movement of the fluid particle represented by the vorticity, for the rotational balance at the vortex centre there is a minimum of pressure, thus the gas density decreases in the vortex core. The density fields of Figure 8.19 High speed interferograms and gas density field reconstruction. $\Delta \mathrm{t}=50 \mu \mathrm{s}-\mathrm{M}=0.4$. show the vortices as concentric rings and confirm that the density decreasing toward the centre. The vortices pass through a formation phase where the density decreases in the vortex centre and a dissipation phase where the density and the size of the vortex both increase. 
Figure 8.19 High speed interferograms and gas density field reconstruction. $\Delta \mathrm{t}=50 \mu \mathrm{s}-\mathrm{M}=0.4$.

\subsubsection{Application to hypersonic flows}

In a hypersonic wind tunnel, the difficulty in measuring gas density is due to the fact that the index variations to be measured in the test section are very small. There are two reasons for this. Firstly, the light deviations are produced by beams that are passing through a phenomenon that is axisymmetric, and not two-dimensional. The integration paths travelled by the beams are therefore shorter, especially in the close vicinity of a shock. Secondly, since the free stream mass density is typically low in such wind tunnels (on the order of $2.10-2 \mathrm{~kg} / \mathrm{m} 3$ ), the index variations caused by the mass density gradients in the passage through the shock waves will induce index variations that remain very small [21]. The example presented here consists of the hypersonic flows around slender axisymmetrical bodies with the purpose of comparing the gas density distributions obtained in the shock layers with the analytical solutions [32]. Here, the objective of the measurement is the radial distribution of the gas density in the shock layer that develops around a cone of length $L=300 \mathrm{~mm}$ and apex semi-angle $\theta_{e}=11.5^{\circ}$. For information, the maximal height of the shock layer is about $13 \mathrm{~mm}$. In theses conditions, a Wollaston prism 
which separates the partial beams very widely has been used. The prism angle itself is about $18^{\circ}$ so that the distance between the two partial beams is $14.60 \mathrm{~mm}$ at the level of the spherical mirror, and it is therefore greater than the domain to be measured. This is equivalent to an ordinary interferometry arrangement with a separate reference, because the interferogram is directly interpretable in a two-dimensional flow but the setup is still differential because one of the beams, used as a reference, is placed in the undisturbed flow, either upstream of the model or, as in the present case, on the side of the model, outside of the shock layers. The interference fringes are placed horizontally so that the separation between the two beams is vertical (Figure 8.20). The results are in good agreement with the analytical solutions on slender ogives following [22] and tabulated data for an inviscid flow of ideal gas around a cone [23].

Figure 8.20 Interferogram and gas density distribution.

\subsubsection{Application to axisymmetric flows}

In the case of axisymmetric wake flows, differential interferometry has been used to analyze the structure of a hot supersonic jet at Mach 1.8 injected into a coaxial supersonic flow at Mach 1.5. The method is sufficiently sensitive for a quantitative analysis to reconstruct the local density field. This operation is possible from a single interferogram provided the flow is twodimensional or axisymmetric. The flow structure was assumed axisymmetric and the 
interferograms were recorded with horizontal fringes, so that vertical gradients of the refractive index are detected [24]. The radial density distribution was determined by spectrum analysis of the colors in the upper half-plane. Wherever possible, a similar analysis was made for the lower half-plane to see whether the flow was effectively axisymmetric. The axisymmetric flow was reconstructed from the undisturbed flow starting as close as possible to the axis of revolution. Figure 8.21 shows two interferograms recorded for two different pressure ratios of 2.74 and 3.38 at the same temperature ratio of 1.67. In the analysis of the interferograms, the density distributions are computed along vertical lines. Whenever the vertical line, where the analysis is performed, completely crosses the jet, a density profile can be determined by integration in the upper half-plane and in the lower-plane. If the flow is strictly axisymmetric, the two profiles should be identical. In Figure 8.21 and for the axisymmetric case, the profiles of the optical thickness (blue line) and the gas density (red line) obtained near the nozzle exit section are relatively symmetric around the flow axis. In the non-axisymmetric case, the analysis made far downstream provides relatively contrasted density profiles which reflect the turbulent behaviour of the flow. 
Figure 8.21 Interferograms of the jet and radial distribution of the gas density.

\subsubsection{Application to gaseous mixture}

Differential interferometry has also been used to analyze the stability of the interface separating two fluids of highly different densities (such as sulphur hexafluoride SF6 and air, or xenon and air) when it is impacted by an incoming shock wave [25]. The shock tube is vertical in order to keep the interface stable before the arrival of the shock wave. In this test, differential interferometry is compared to an other diagnostic technique based on densitometry where the partial gas density profile of one of the two gases can be obtained with careful calibration if the gas pair is air/xenon. In the case of SF6/air, densitometry technique cannot be used because both gases are transparent to this technique. Only differential interferometry can yield a measurement of SF6 distribution in air. The optical setup is that shown in Figure 8.10. It requires two Wollaston prisms $\left(0.5^{\circ}\right.$ bonded angle $)$ installed head to foot and two "Clairaut" achromatic lenses, $800 \mathrm{~mm}$ in focal length and $120 \mathrm{~mm}$ in diameter. In the case of two-gas mixtures, it is known that the Gladstone-Dale relation can be extended if the Gladstone-Dale constants of each 
gas are known [1]. Then, the analysis of the interferogram yields the partial density profile of one of the two gases across the interface. Figure 8.22 shows three interferograms recorded at different times. On the interferogram (a), the shock wave has already crossed the interface, has been reflected from the tube end wall and is about to again impact on the modified interface. Picture (b) was taken shortly after this second impact and the wave is seen to have been partly transmitted into SF6 and partly reflected into the air. On picture (c) the transmitted wave can be seen close to the bottom of the picture while the reflected part has again been reflected from the end wall and is about to impact on the interface.

The SF6 partial density profiles were obtained through the interface by averaging a dozen interferograms. For the xenon/air gas pair, the xenon partial density profiles were compared to those obtained by densitometry technique. The two techniques yield very similar results.

Figure 8.22 Gas density profile of SF6 - Interface SF6-Air, $M_{s}=1,45$.

\subsection{Principles of color holographic interferometry}

The use of differential interferometry implies data integration to get the full gas density and this integration results in a certain measurement inaccuracy. To obtain absolute data, realtime true color holographic interferometry using a three-color laser source has been developed [26]. The various holographic interferometry methods include double exposure, time-averaged, 
or real-time holography. Until recent years, experiments in holographic interferometry were performed with a single laser, i.e., they were monochromatic. Most experiments found in the literature relate to transmission holograms [3] and few experiments have been performed to date using holographic interferometry with reflected white light [27],[28]. It should be said that, in monochromatic mode, experiments in reflected white-light holography have little advantage over holographic interferometry in transmitted light. Some publications mention the use of threewavelength differential interferometry [17] and holographic interferometry by reflection [29],[30] and all show that the essential advantage of colour is that the achromatic fringe can be located in the observed field.

\subsubsection{Principle of transmission and reflection holograms}

Real-time true colour holographic interferometry uses three primary wavelengths (red, green, blue) to record the interference between the three object beams and the three reference beams simultaneously on a single reference hologram. The technique uses a single-layer silverhalide panchromatic plate made of a gelatine film with immersed silver grains, sensitive in the range $450-690 \mathrm{~nm}$. The size of silver grains of the gelatine is about $10 \mathrm{~nm}$ and the gelatine thickness is of the order of $10 \mu m$. Under no-flow conditions, the undisturbed object red, green and blue waves $\sum R O, \sum G O$ and $\sum B O$ are recorded in the hologram by virtue of their interference with the three reference waves $\sum R R, \sum G R$ and $\sum B R$. As it can be seen in Figure 8.23 , step 1 , for a plate recorded in transmission, the three reference waves and the three object waves arrive on the same side of the plate while in reflection, they come from opposite sides of the holographic plate. After treatment of the plate and resetting in the optical bench (Step 2), the three reference waves $\sum R R, \sum G R$ and $\sum B R$ are diffracted by transmission or by reflection 
according to the recording mode used to form the three diffracted object waves $\sum R O D, \sum G O D$ and $\sum B O D($ Step 3, Figure 8.23).
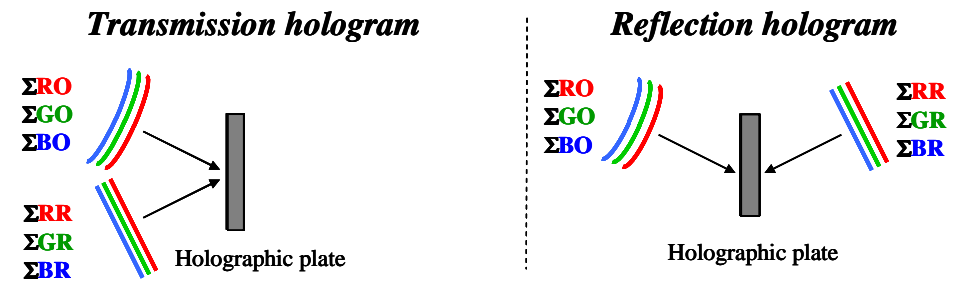

1) Recording : $1^{\text {st }}$ exposure,

2) Development of hologram and resetting
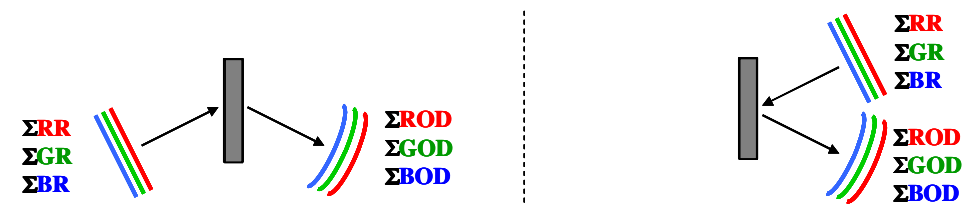

3) Reconstruction with only the three reference waves
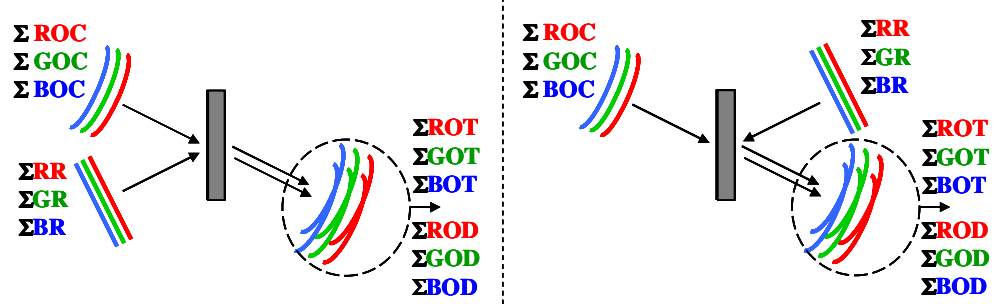

4) Reconstruction with the three reference waves and the three undisturbed object waves

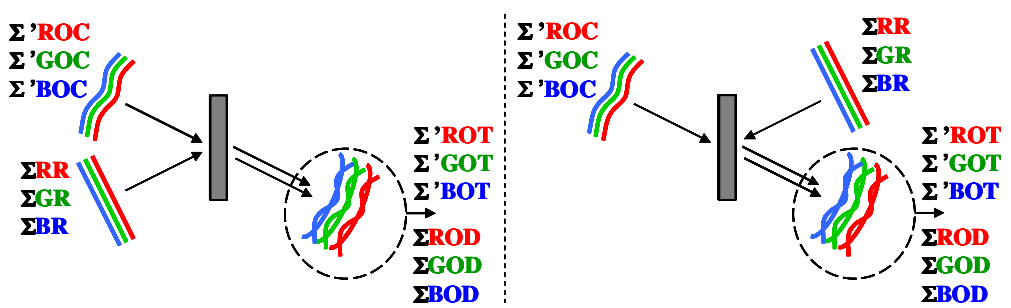

5) Reconstruction with the three reference waves and the three disturbed object waves

Figure 8.23 Formation of colors interferences fringes

Then the hologram is illuminated simultaneously by the three reference beams and three object beams, from which we get the three object beams waves $\sum R O D, \sum G O D$ and $\sum B O D$ reconstructed by the holographic plate simultaneously with the three live object waves transmitted waves $\sum R O T, \sum G O T$ and $\sum B O T$. The profiles of the $\sum R O D$ and $\sum R O T, \sum G O D$ 
and $\sum G O T$ waves, and the $\sum B O D$ and $\sum B O T$ waves are strictly identical to each other if no change has occurred between the two exposures (no flow in the test section) and if the hologram gelatine has not contracted during development. So there will be three simultaneous interferences among the object waves constructed by the hologram and the live object waves. In this case, a flat uniform colour can then be observed behind the hologram (Step 4). If a change in optical path is created in the test section of the wind tunnel, the three live waves will deform and adopt the profiles $\Sigma^{\prime} R O T, \Sigma^{\prime} G O T$ and $\Sigma^{\prime} B O T$ while the waves reconstructed in the hologram, $\sum R O D$, $\sum G O D$ and $\sum B O D$, remain unchanged. Any colour variations representing optical path variations will thus be visualized in real time behind the hologram (Step 5, Fig. 8.23).

\subsubsection{Optical setups of real-time holographic interferometry}

\subsubsection{Real-time color transmission interferometer}

An example optical setup is shown in Figure 8.24. The three wavelengths downstream of the acousto-optic cell are split into a reference beam and object beam by a beam splitter cube. A right angle prism is used to adjust the reference and object path lengths on the hologram. A spatial filter is used to expand the beam for its passage through the test section. A pair of achromatic lenses converts the beam into parallel light in the test section and then focuses it on the hologram. The reference beam passes over the test section, and then another achromatic lens is used to illuminate the hologram with a parallel light beam. In order to provide a feeling of the capability of the technique and the illumination power involved, an example of implementation of this experimental setup is given in [34]. In their application the object beam diameter is $40 \mathrm{~mm}$ at the hologram and that of the reference beam is $60 \mathrm{~mm}$. At the acousto-optic cell, the power of the three light waves is practically the same (of the order of $70 \mathrm{~mW}$ per channel). The 
beam splitter cube distributes $85 \%$ of this power to the reference path and $15 \%$ to the measurement path.

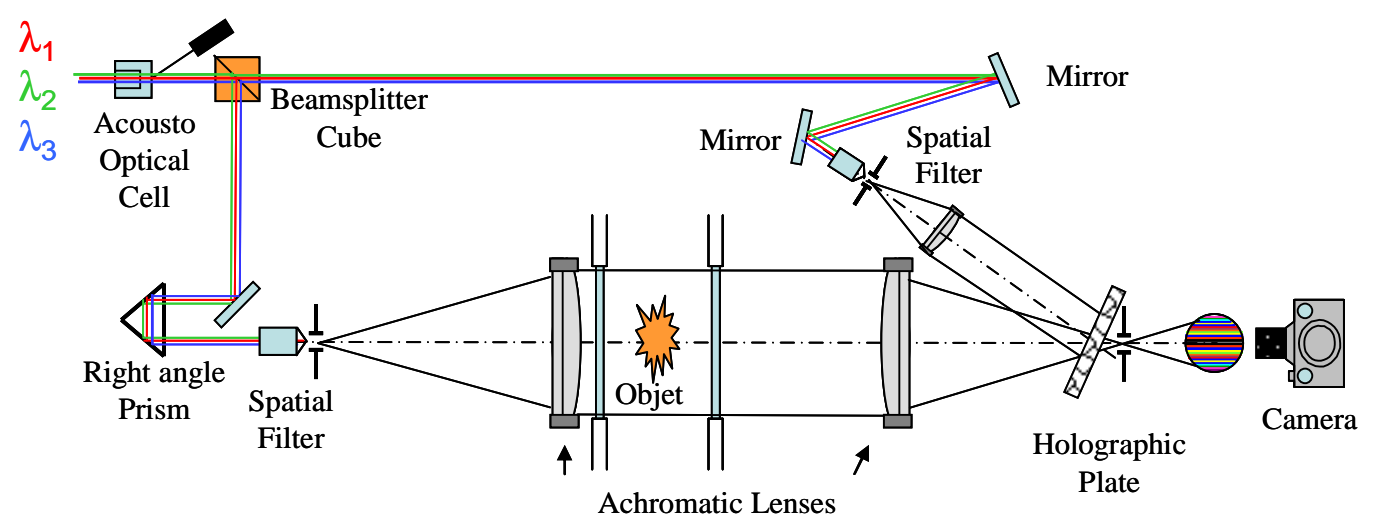

Figure 8.24 Optical setup implemented around the wind tunnel.

At the hologram, [34] measured $250 \mu \mathrm{W} / \mathrm{cm}^{2}$ in the red and blue lines and $280 \mu \mathrm{W} / \mathrm{cm}^{2}$ in the green line for the reference beam, while the object beam powers are $30 \mu \mathrm{W} / \mathrm{cm}^{2}$ in the red line and $40 \mu \mathrm{W} / \mathrm{cm}^{2}$ in the green and blue lines. These proportions can be used to obtain a perfect balance among the powers of the three waves diffracted by the hologram when re-positioning it, and the three live waves. For reference, in the proposed setup the hologram diffracts $70 \mu \mathrm{W} / \mathrm{cm}^{2}$ in the red line, $65 \mu \mathrm{W} / \mathrm{cm}^{2}$ in the green line, and $90 \mu \mathrm{W} / \mathrm{cm}^{2}$ in the blue line. The holograms are then subjected to treatments to harden the gel, develop it, and bleach it. When the hologram is put back in place, the light power at the camera entrance is $1.510^{-3} \mathrm{~W}$ at the focal point, which is sufficient to record interferograms at an ultra-high speed of 35,000 frames per second with an exposure time of $750 \mathrm{~ns}$ per shot.

\subsubsection{Real-time color reflection interferometer}

The optical setup of Figure 8.25 could be named "Denisyuk" because it uses a holographic plate 
in a classical Lippmann-Denisyuk in-line experiment. To obtain a very simple setup, all the optical pieces are located on the same side of the wind tunnel, except the flat mirror which reflects the light rays back into the test section. Due to these considerations, the optical setup shown in Figure 8.25 based on real-time colour reflection holographic interferometry has been designed.

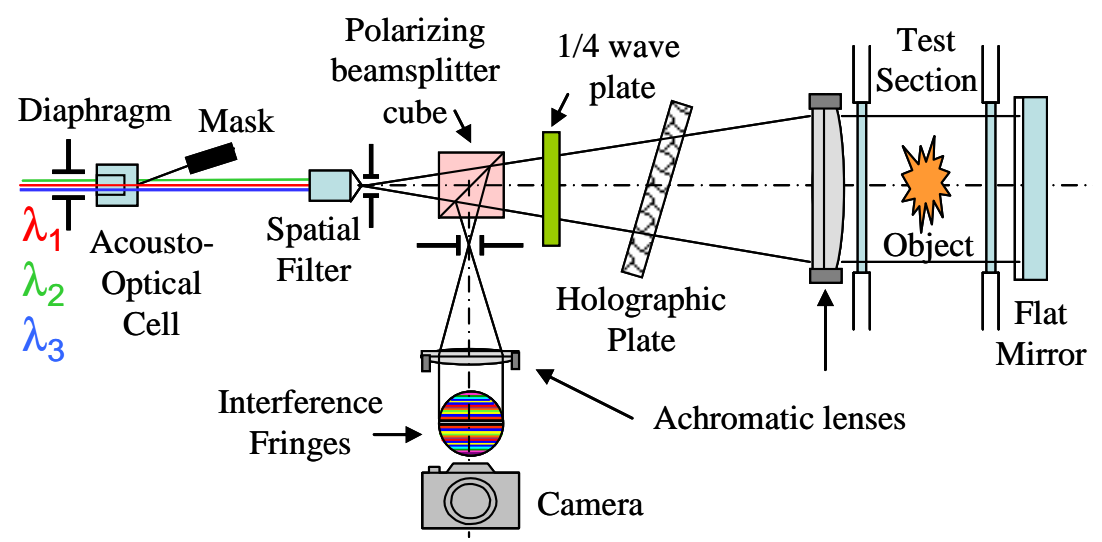

Figure 8.25 Real-time three-color reflection holographic interferometer.

The light source used behind the interferometer can be constituted with three different lasers: a red line $\left(\lambda_{1}=647 \mathrm{~nm}\right)$, a green line $\left(\lambda_{2}=532 \mathrm{~nm}\right)$ and a blue line $\left(\lambda_{3}=457 \mathrm{~nm}\right)$, for example. A flat mirror located just behind the test section returns the three beams on the holographic plate inserted between the quarter wave plate and the large achromatic lens. The hologram is illuminated on the two sides by the three collimated reference and measurement waves which are formed by the convergent and divergent achromatic lenses (not shown in Figure 8.25). This arrangement allows one to easily obtain before the test a uniform background colour (infinite fringes) or narrowed fringes (finite fringes). In this setup, a polarizing beam splitter cube is inserted between the spatial filter and the quarter wave plate which transforms the waves polarization twice (from $\mathrm{P}$ parallel to circular and from circular to $\mathrm{S}$ parallel) so that, when the 
rays are returning, the beam splitter cube returns the rays towards the camera. A diaphragm is placed in the focal plane just in front of the camera in order to filter out any parasitic interference. The interferences fringes produced by the phenomenon under analysis can be directly recorded using high speed camera. Figure 8.26 details how the interferences fringes are generated in the real-time three-color reflection holographic interferometer.

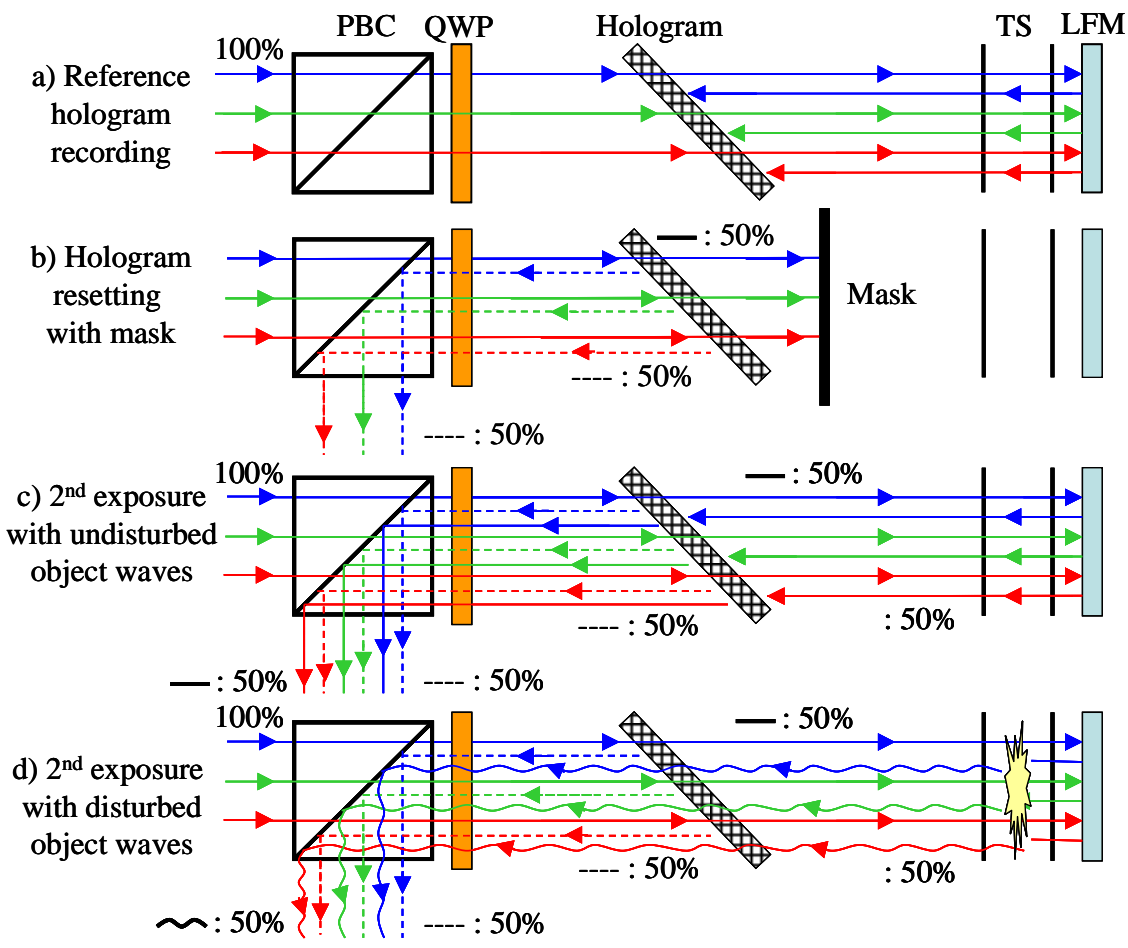

Figure 8.26 Formation of colour interference fringes in optical setup.

First, the holographic plate is simultaneously illuminated with the three wavelengths

(Figure 8.26a). The panchromatic hologram records simultaneously the three sets of interference fringes produced by the three incident waves and the three waves reflected by the flat mirror (first exposure). Then the hologram is developed and it is reset in the optical bench at the same location. At the second exposure, if the diffraction efficiency of the holographic plate is near to $50 \%$ for the three lines, $50 \%$ of the light is reflected by the hologram (dashed lines) and $50 \%$ 
crosses the holographic plate (solid lines). If a mask is inserted in the front of the test section, one can observe on the screen the three images diffracted by the plate (Figure 8.26b). This operation allows for verifying the quality of the holograms diffracted. When the mask is moved, $50 \%$ of the light crosses the test section twice and interferes in real-time with the three references waves (solid lines). Interference fringes are not localized because they can be observed from the holographic plate to the camera. If no disturbances exist in the test section, a uniform background colour is obtained in the camera (Figure 8.26c). If variation in refractive index exists in the test section, colour fringes will be seen on the screen. As the luminous intensities of reference and measurement waves are basically equal, the contrast of colour interferences fringes will be maximum (Figure 8.26d).

This optical setup is very simple but it presents some advantages and some inconveniences. The advantage lies in the small number of optical pieces which are used. The reference beams and the measurement beams are co-linear and there is just a flat mirror behind the test section. The contrast of colour interferences fringes depends on the diffraction efficiency of the holographic plate and the colours saturation depends on the luminous intensity of the three wavelengths which can be adjusted with the acousto-optic cell. A main inconvenience resides in the fact that it is not possible to adjust the diffraction efficiency of the holographic plate. It is only fixed by the chemical treatment and it is a function of gelatine thickness. The unique solution to solve this problem will consist in a specific treatment of the surface of the flat mirror and this operation implies prior knowledge of the diffraction efficiency of the hologram. 
Finally, the three interference fringe patterns will exist and can be recorded if the coherence length of the three wavelengths is more than twice the distance between the holographic plate and the flat mirror located just behind the test section. Compared to the setup of transmission holographic interferometry, here it is not possible to adjust the length between the reference and measurement rays.

\subsubsection{Problem of gelatine contraction}

The problem of gelatine shrinkage is described in detail in [33]. In Figure 8.27, one can see how the interference fringes are inscribed into the gelatine when the holographic image is recorded by transmission or reflection. In transmission, the interference fringes are perpendicular to the plate and a small variation in the gelatine thickness caused by the chemical treatment of the hologram does not modify the three inter fringe distances. On the other hand, in reflection, interference fringes are recorded parallel to the plate surface and the inter fringe distance is very sensitive to a small variation of the gelatine thickness. Figure 8.27 presents the effects of the gelatine contraction when a reflection hologram is recorded with a green wavelength $(514 \mathrm{~nm})$. During the reconstruction, a white light source (for example a xenon source) illuminates three different holograms at the incidence angle that the reference wave had at recording. One can see that if the gelatine thickness is kept constant $(\Delta e=0)$, the hologram only diffracts the recording wavelength, i.e. for the green hologram, the green wavelength contained in the xenon spectrum. If the gelatine thickness has decreased by $5 \%,(\Delta e=-0.5 \mu \mathrm{m}$ for a $e=10 \mu \mathrm{m}$ gelatine thickness), the fringe spacing will be proportionally reduced and the diffracted wavelength will be shifted by a quantity equal to: 


$$
\Delta \lambda=\frac{\lambda}{e} \Delta e
$$

The hologram will diffract a wavelength equal to $488.3 \mathrm{~nm}$ corresponding to a blue line for a $\Delta e=-0.5 \mu m$. If the gelatine thickness increases by $10 \%(\Delta e=+1.0 \mu m)$, the hologram illuminated in white light will diffract a wavelength close to yellow $(565.4 \mathrm{~nm})$.
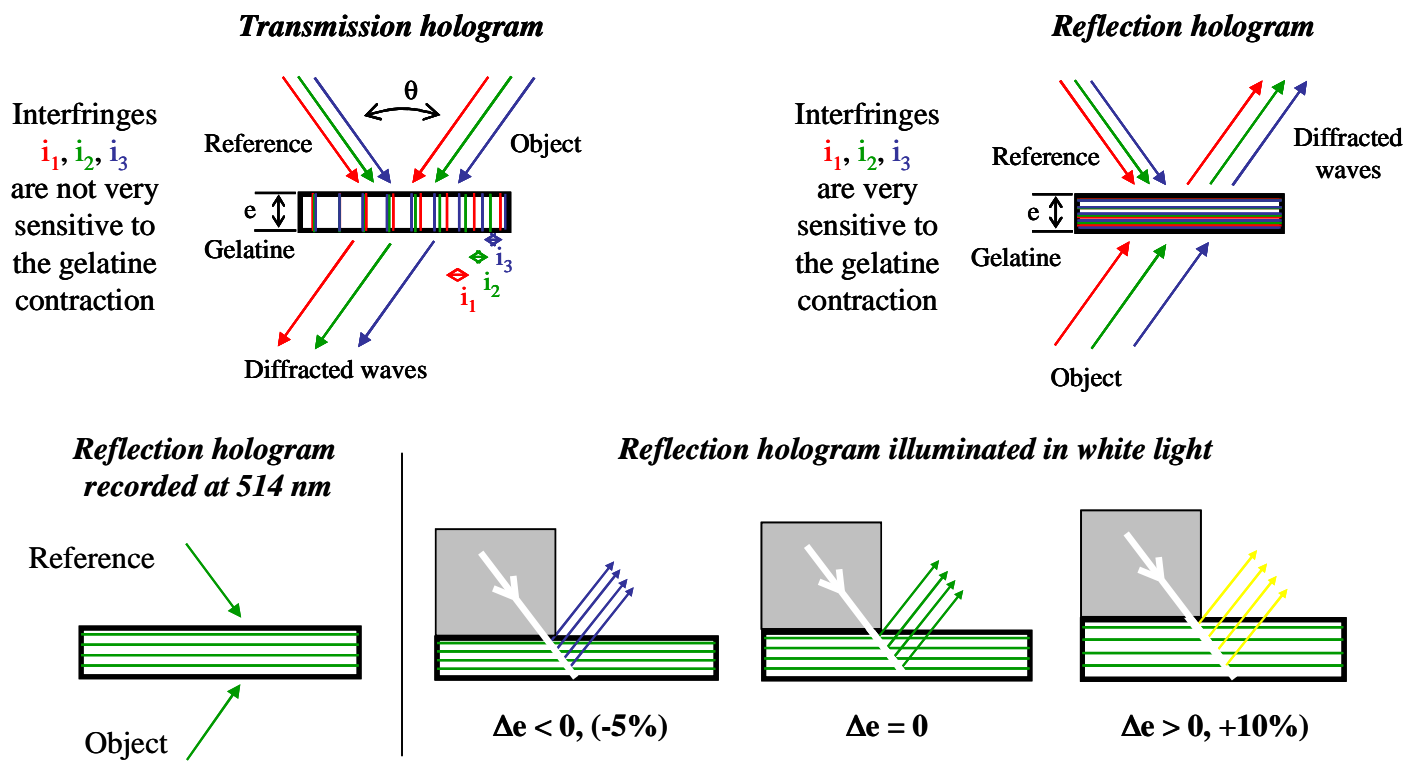

Reflection hologram illuminated in white light

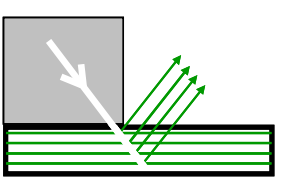

$\Delta \mathrm{e}=\mathbf{0}$

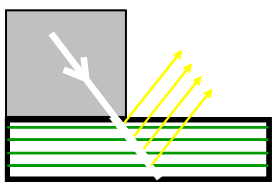

$\Delta \mathrm{e}>\mathbf{0},+\mathbf{1 0 \%})$

Figure 8.27 Effect of the gelatine contraction on the different waves.

On the other hand, it is well known that the chromatic perceptibility of eye $\delta l$ varies with the wavelength. The chromatic perceptibility is the variation $\delta l$ between two different wavelengths perceived by the eye at constant luminosity. It is about $1 \mathrm{~nm}$ in the green and yellow colours and $6 \mathrm{~nm}$ in the blue and red colours, which corresponds to relative variations of $0.2 \%$ and $1.5 \%$ respectively. For the diffracted colour change not to be detected by a human eye, it is mandatory that $\delta l / \lambda$ has to be less than $\delta \lambda / \lambda$ that which implies that the variation in gelatine thickness should be less than $0.2 \%$. This means changes in thickness of more than $20 \mathrm{~nm}$ are not acceptable. As the optical technique is based on the knowledge of the true colours diffracted by 
the hologram, variations of the gelatine thickness are a cause of large errors in the data analysis. It is for this reason that the gelatine shrinkage problem has to be perfectly mastered. Details of different holographic plates used (PFG03c from Slavich and Ultimate08 from Gentet), the solution to control the gelatine contraction and the measurements of diffraction efficiency can be found in [31].

\subsubsection{Applications}

\subsubsection{Transmission interferometer}

The optical setup of real-time transmission holographic interferometry has been applied to analyse the unsteady flow downstream of a cylinder with diameter $D=20 \mathrm{~mm}$ in crossflow. Figure 8.28a gives a sequence of six interferograms of the flow around the cylinder at Mach 0.37. The time interval between each picture is $100 \mu \mathrm{s}$. One can see that each vortex is represented by concentric rings of different colours with each colour representing an isochoric line. The vortex formation and dissipation phases can be visualized clearly by the fringes oscillation between the upper and lower surfaces of the cylinder. Several types of measurements were made by analyzing a sequence of 100 interferograms. First, the vortex centre defined by the centre of the concentric rings was located in space for each interferogram, which made it possible to determine the mean paths for the vortices issuing from the upper and lower surfaces. The results of this are shown in Figure 8.28b. The "o" symbols represent the positions of the vortex centres from the upper surface, and the "•" symbols those of the lower surface.

Remarkably, the two paths exhibit a horizontal symmetry about the $x=0$ axis passing through the cylinder centre. One may also point out that even at $x / D=4$ downstream of the cylinder, the upper and lower vortex paths do not come together and line up. Then, the colours of each interferogram were analyzed using a modelling of the luminous intensity of interference fringes 
versus the optical path difference called "MIDI" software, which models the light intensity and experimental interference fringe colours as a function of the path difference [9]. The gas density measured under free stream conditions is the same as that measured at the outer flow of the wake (measured in the vicinity of the wind tunnel's upper and lower walls).

Figure 8.28 Unsteady wake flow around the cylinder -Results and analysis - Mach 0.37

The graph of Figure 8.28c shows the variations of $\rho / \rho_{0}$ varies for the vortices emanating from the upper and lower surfaces. The trend curves plotted show the same variations. For $0.5<$ $x / D<1$, the vortices are in a formation or agglomeration phase because the gas density decreases at their centre. Then, when $x / D>1$, the vortices enter a dissipation phase because the gas density increases again at their centres. The drop in gas density is large, reaching about $20 \%$ of $\rho_{0}$. A rather large dispersion may nonetheless be noticed in the data. This is due mainly to the uncertainty in determining the vortex centre locations, which is not trivial when the vortices are in the dissipation phase. 


\subsubsection{Reflection interferometer}

A real-time colour reflection holographic interferometer has been implemented to analyse the same unsteady wake flow around the cylinder. In this experiment, the infinite Mach number was fixed at 0.45 and the high speed interferograms were recorded with the rotating drum camera which is equipped with a 400ASA colour film. The time interval between two successive frames is $117 \mu s$ and the time exposure is $750 n s$. Several movies have been recorded with uniform background colour (infinite fringes), circular and narrowed fringes (finite fringes). As the optical setup is very sensitive to external vibrations, the uniform background colour is difficult to adjust when the wind tunnel is running, but the fringe formation can be observed on the hologram surface so that it is possible to adjust the uniform background colour with the wind tunnel operating. Figure 8.29 shows three of twelve interferograms covering about a period of the vortex street. They are recorded in infinite fringes. The interferogram colours are well saturated and of higher contrast than those obtained in previous experiments performed with transmission holograms (see the interferograms of Figure 8.27).

When the background colour is uniform, it is very easy to follow the vortices emitted from the upper and lower side. For instance, if one looks at the colours coming out in the vortex cores, one can easily see that the first vortex emitted from the upper side enters a formation phase where the gas density decreases in the vortex centre. A second phase of dissipation is observed looking at the last vortex leaving the observed field. Finally, as with transmission holographic interferometry, each colour represents a value of the gas density. In the post-processing analysis, the gas density field was presented in non-dimensional form with respect to $\rho_{0}$, the stagnation gas density. One can see that the instantaneous gas density varies from 0.70 to 0.98 . The average 
gas density in the field has been calculated from twelve successive interferograms. The interferogram number is not very significant, but the obtained field is already symmetrical enough and the gas density varies from 0.72 to 0.97 . Finally, if the colour scale of interference pattern is very well known to the user, the image of interferograms is sufficient to correctly evaluate the evolution of the gas density field.

Figure 8.29 Interferogram analysis : instantaneous and average gas density fields

\section{References}

[1]. Merzkirch, W. 1974. Flow visualization. New-York: Academic Press.

[2]. West, C.M. 1978. Holographic interferometry. New-York: Wiley-Interscience.

[3]. Rastogi, P.K. 1994. Holographic interferometry. Berlin: Springer-Verlag.

[4]. Kreis, T. 1996. Holographic interferometry, Akademie Verlag.

[5]. Mercer, C. 2003. Optical metrology for fluids, combustion and solids, Kluwer Academic Publishers.

[6]. Fleury, P., and Mathieu, J.P. 1960. Images optiques, Interférences. Editions Eyrolles.

[7]. Veret, C., Philbert, M., Surget, J., and Fertin, G. 1977. Aerodynamic flow visualization in the ONERA facilities. ISFV1, Asanuma: Hemisphere. 
[8]. Dewey, J.M., Heilig,W., Reichenbach, H., and Walker, D.K. 1983. The analysis of coloured interferograms of shock waves. Flow visualization I; Washington: Hemisphere.

[9]. Desse, J.M. 1997a. Recording and processing of interferograms by spectral characterization of the interferometric setup. Exp. Fluid 23:265-71.

[10]. Gontier, G. 1957. Contribution à l'étude de l'interférométrie différentielle à biprisme de Wollaston. Pub. Sci. Tech. Ministère de l'air 338.

[11]. Françon, M., and Sergent, B. 1955. Compensateur biréfringent à grand champ. Opt. Acta:182-4.

[12]. Smeets, G. 1968. Interféromètre differential à faisceaux fortement séparés. Dépouillement des interférogrammes. ISL TN, 41.

[13]. Carlomagno, G.M. 1986. A Wollaston prism interferometer used as a reference beam interferometer. Flow visualization IV, Washington: Hemisphere.

[14]. Desse, J.M. 1993. Direct measurement of the density field using high speed differential interferometry. Exp Fluid 15:452-8.

[15]. Desse, J.M., and Picart, P. 2013. Digital three-wavelength holographic interferometry using Wollaston prisms. Digital Holography \& 3D Imaging, OSA Meeting, Hawaï.

[16]. Desse, J.M. 1990. Instantaneous density measurement in two dimensional gas flow by high speed differential interferometry. Exp fluid 12:1-9.

[17]. Desse, J.M. 1997b. Three-color differential interferometry. Appl. Opt. 36:7150-6.

[18]. Mac Adam, D.L. 1985. Color measurement, Theme and Variations. Springer-Verlag, New-York, 27.

[19]. Desse, J.M. 2003. Oil-film interferometry skin-friction measurement under white light. AIAA J. 41:2468-77.

[20]. Sieverding, C.H., Cicatelli, G., Desse, J.M., Meinke, M., and Zunino, P. 1999; Experimental and numerical investigation of time varying wakes behind turbine blades. Notes on numerical fluid mechanics. 67:Vieweg.

[21]. Desse, J.M., and Deron, R. 2009. Shadow, schlieren and color interferometry. Aerospace Lab. 1:1-10.

[22]. Merlen, A., and Andriamanalina, D. 1992. Analytical solutions for hypersonic flow past slender power law bodies at small angle of attack. AIAA J. 11. 
[23]. Jones, D.J. 1969. Tables of inviscid supersonic flow about circular cones at incidence $\gamma=1.4$, Agardograph 137.

[24]. Rodriguez, O., Desse, J.M., and Pruvost, J. 1997. Interaction between a supersonic hot jet and a coaxial supersonic flow. Aero. Sci. Technol. 6:369-79.

[25]. Galametz, I. 1994. Visualisation et mesure de masse volumique dans un mélange gazeux en tube à choc. Thèse de doctorat, Université de Lille.

[26]. Desse, J.M., Albe, F., and Tribillon, J.L. 2002; Real-time color holographic interferometry. Appl. Opt. 41:5326-33.

[27]. Smigielski, P., Fagot, H., and Albe, F. 1976. Application de l'holographie ultra rapide à référence arrière à l'étude de déformations dynamiques, Proc. 12th International Congress of High Speed Photography, Toronto.

[28]. Vikram, C.S., and Witherow, W.K. 1992. Critical needs of fringe order accuracy in twocolour holographic interferometry", Exp. Mechanics. 74-7.

[29]. Harthong, J., Sadi, J., Torzynski, M., and Vukicevic, D. 1997. Speckle phase averaging in high-resolution color hologhraphy, J. Opt. Soc. Ame. A. 14:405-10.

[30]. Jeong, T.H., Bjelkhagen, H.I., and Spoto, L.M. 1997. Holographic interferometry with multiple wavelengths, Appl. Opt. 36:3686-8.

[31]. Naydenova, Y. 2011. Advanced holography-Metrology and imaging. InTech Open Acces Publisher.

[32]. Desse, J.M., and Fabre, E. 1996. Differential interferometry for studying hypersonic flows. Exp. Fluid 20:273-8.

[33]. Desse, J.M. 2006. Recent contribution in color interferometry and applications to highspeed flows. Opt. Lasers Eng. 44:304-20.

[34]. Desse, J.M., and Picart, P. 2012. Color holographic interferometry (from holographic plates to digital holography). 15th International Symposium on Flow Visualization June 25-28, 2012, Minsk, Belarus.

\section{Problems}

1) An optical setup based on differential interferometry with double crossing of the test 
section and one Wollaston prism is used to analyze a field of $200 \mathrm{~mm}$ in diameter. It includes a spherical mirror, $400 \mathrm{~mm}$ in diameter, 2,5 meter in the radius of curvature. We want to realize an absolute measurement of the optical path difference. Knowing the quartz birefringence $\left(n_{e}-n_{0}\right)$ is equal to $9.10^{-3}$ and the calcite birefringence is 20 times higher than quartz, determine the bonding angle of the prism if the material is calcite or quartz. What material should I use? Why?

\section{Solution 1:}

- The distance between the two interfering rays is $d x=\varepsilon \cdot R=200 \mathrm{~mm}$.

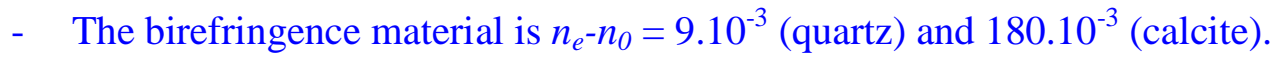

- The bonding angle is given by $\alpha=\arctan \left(\frac{d x}{2 R\left(n_{e}-n_{0}\right)}\right)=77^{\circ}$ for quartz and $12.5^{\circ}$ for calcite.

- It is not possible to build a quartz Wollaston because the bonding angle is too large. The unique solution is to build a calcite Wollaston.

$* * * * * * * * * * * * * * * * * * * *$

2) A two-dimensional flow is analyzed with the differential interferometer defined in question (1). The test section located at $100 \mathrm{~mm}$ from the spherical mirror is $50 \mathrm{~mm}$ in width. The flow generates a negative optical path difference of 0,55 microns. What is the decreasing of gas density measured in the test section? Without the flow, the differential interferometer is adjusted to obtain a white color on the screen $(\delta=0)$. When the wind tunnel is running, what is the color induced by the flow?

\section{Solution 2:}

$-\delta$ is the optical path difference $(0.55 \mu \mathrm{m})$ with $\delta=2 . E$ because the phenomenon is crossed twice by the luminous rays. Equation (8.25) gives E: the optical thickness equal to 
K.e. $\rho / \rho_{s} . \rho_{s}$ is the gas density under standard conditions $\left(1\right.$ atmosphere, $\left.0^{\circ} \mathrm{C}\right)$.

$$
\Delta \rho=\frac{E . \rho_{s}}{K . e}=\frac{0.55 \cdot 10^{-6} * 1.293}{296 \cdot 10^{-6} \cdot 50.10^{-3}}=-48.10^{-3} \mathrm{~kg} / \mathrm{m}^{3}=-48 \mathrm{~g} / \mathrm{m}^{3}
$$

- From Equation 8.18, $\delta=\Delta=2 . e .\left(\xi-\xi_{0}\right) . \xi_{-} \xi_{0}$ is equal to $0.355 \mathrm{~mm}$. According to the prism calibration shown in Figure 8.11, the colour is blue.

$$
* * * * * * * * * * * * * * * * * * * *
$$

3) What is the space fringe recorded in a transmission hologram $(\lambda=660 \mathrm{~nm})$ if the two interfering rays are separated by $30^{\circ}$. How many fringes are recorded in the gelatine thickness $(10 \mu \mathrm{m})$ ? What is the effect of gelatine contraction on the space fringe?

\section{Solution 3:}

- the fringe space (interfringe) is given by :

$$
-i=\frac{\lambda}{2 \sin (\theta / 2)}=\frac{0.66}{2 * 0.259}=1.28 \mu m
$$

- Fringes are recorded perpendicular to the plate (the fringes number depends on the hologram surface).

- no effect in transmission

$* * * * * * * * * * * * * * * * * * * *$

4) What is the space fringe recorded in a reflection hologram $(\lambda=660 \mathrm{~nm})$ if the two interfering rays are separated by $155^{\circ}$. Before the development, the gelatine thickness is decreased by $5 \%$. What wavelength is diffracted by the hologram if it is illuminated in white light?

\section{Solution 4:}

$$
-i=\frac{\lambda}{2 \sin (\theta / 2)}=\frac{0.66}{2 * 0.86}=0.38 \mu m
$$


- Fringes number : $10 / 0.38=26.3$ fringes

- According to relationship 8.40:

$\Delta \lambda=0.66 \frac{0.5}{10}=0.033 \mu m$

the wavelength diffracted is equal to : $0,660-0.033=0.627 \mu \mathrm{m}$

5) $* * * * * * * * * * * * * * * * * * * *$ In a hypersonic wind tunnel, test air density is equal to $2.10^{-2}$

$\mathrm{kg} / \mathrm{m}^{3}$ and the test section width is $15 \mathrm{~cm}$. Considering density variations up to $300 \%$, quantify the refractive index variations for a plane $2 D$ experiment.

\section{Solution 5:}

- $\quad$ By using the relationships 8.28 and $E=(n-1) \cdot e$ :

$$
\frac{\rho}{\rho_{0}}=3=\frac{E}{E_{0}}=\frac{(n-1) \cdot e}{\left(n_{0}-1\right) \cdot e}
$$

$3\left(n_{0}-1\right)=n-1$

- Using Gladstone-Dale relationship, $\left(n_{0}-1\right)=K . \rho_{0} / \rho_{s}=296.10^{-6} * 2.10^{-2} / 1.293=4.57 .10^{-6}$ $\mathrm{n}-1=13.73 .10^{-6}$

and $\mathrm{n} / \mathrm{n}_{0}=1.00001373 / 1.1 .00000457=1.00000916$ 